\title{
Corporate Web Reporting: An Empirical Investigation of Indian Companies Websites Content \& Design Features
}

\author{
Effulgence \\ Vol. 16 No. 2 \\ July - December, 2018 \\ Rukmini Devi Institute of Advanced Studies \\ E-mail : effulgence@rdias.ac.in, Website : www.rdias.ac.in \\ http:/ / effulgence.rdias.ac.in/user/default.aspx \\ https://dx.doi.org/10.33601/effulgence.rdias/v16/i2/2018/57-77
}

\author{
Dr. Arwinder Singh ${ }^{1}$ \\ Harmandeep Singh ${ }^{2} \otimes$
}

\begin{abstract}
Business firms use internet to satisfy the growing needs of their stakeholders. They are disseminating various companies' information on the internet. Therefore, Internet reporting has various benefits to the source company such as wider reach, mass communication, speed, and low cost. The focus of the present study is to examine the extent of corporate web reporting practices across various industries in India. The study is based on 183 BSE listed companies from the database of BT-1000 for the year 2015-16, and these companies are broadly categorized into sixteen sectors. The results reveal that various content and presentation features, such as online chat facility, E-commerce facility, webcasting, animation, hyperlink to the annual report from the homepage and many more are not fully disclosed across industries. It also found that Indian companies have uniformity in the disclosure of companies' mandatory information across the various industry groups. The results of ANOVA indicate that there is the significant difference in the corporate web reporting practices among the Indian companies across different industries groups.
\end{abstract}

Keywords: Web Reporting, Website Content, Website Presentation.

\section{INTRODUCTION}

$T^{3}$ he rapid growth of internet technology, have significantly impacted on the accounting practices. Many of the companies worldwide utilize the internet to disseminate their financial as well as non-financial information on the web (Debreceny et al. 2002). The reporting through the internet is highly accepted by companies all over the world since 1996 (Louwers et al. 1996) because it provides various benefits to source company. Firstly, it helps in reducing costs associated with communication of company information such as printing, postage, delivery, etc. (Lymer, 1999). Secondly, it reaches widely, limitless to content, and cross the territorial boundaries. Thirdly, through web reporting, companies improved their presentation of information by using graphics, animation, navigation, hyperlink, etc. (Garg and Verma, 2010). Lastly, the internet reporting allows the company to get integrated reporting by giving all dimension of

1. Assistant Professor, Department of Commerce, GNDURC, Gurdaspur E-mail: arwinder.gndu@yahoo.com

2. Senior Research Fellow, Department of Commerce, GNDURC, Gurdaspur, E-mail: har90bal@gmail.com 
corporate reporting such as financial, social, and marketing on one platform, i.e., corporate web pages.

Most of the studies on web reporting have been conducted in developed economies such as USA, UK, and Australia during the last decades (see Louwers et al. 1996; Lymer, 1997; Ettredge et al., 2000; Debreceny et al. 2002; Larran and Ginner, 2002) and only a few studies have been conducted in India (see Raman et al., 2003; Singh, 2009; Garg and Verma, 2010). The present study focuses on the extent of internet reporting of different industry groups of listed Indian companies. The recent growth of web-based corporate reporting have shown that that specific industry groups for example industry related to technology, finance may have more incentives to adopt this practice in a broader way than other industries (Bonson and Escobar, 2002). Wallace et al., (1994) justified the idea that organizations corporate disclosure practices are probably going to shift over various industry groups and proposed that the nature or significance of an industry sort to the stakeholders may clarify expected differences in corporate disclosure level across different industries. Hence, the present study examines the extent of corporate web reporting practices of content and presentation attributes across the various industry groups and to test significance of differences in web reporting practices across the various industry groups.

\section{LITERATURE REVIEW AND HYPOTHESIS DEVELOPMENT}

An extensive literature have assessed the extent of internet reporting, Lymer, 1997 investigated 50 UK companies and found $68 \%$ use internet for disseminating financial information. Xiao et al. (1997) analyzed the four dimension of internet reporting, i.e., content and form of financial reporting, the role of the internet (problem solver or creator), the determinant of technology \& nontechnology and pace of change. Carven and Marston (1999) examined the extent of financial information disclosure on the internet by UK companies. Debreceny and Gray (1999) studied financial reporting on the internet and found companies using internet technologies such as PDF, HTML for presenting the information. Ettredge et al. (2000) analyzed the accounting content of 100 companies and found that it was mostly providing accounting information such as balance sheet, cash flow, and income statement. Larran and Ginner (2002) studied the use of the internet for corporate reporting by 144 Spanish companies and found that 107(74\%) companies had websites and of the sample companies 67\% IBEX and 50\% IGBM included annual accounts on their web pages. This study reveals a statistically significant association between the size of the company and extent of corporate disclosure on the internet. Raman et al. (2003) survey 32 leading Indian corporate sector reporting practices through the web. The analysis of result reveals that $87.5 \%$ (28) companies had a website and $46.43 \%$ (13) companies of them presented some financial information on their web pages. Garg and Verma (2010) studied the 200 BSE listed Indian companies and found all the companies had the website with more than $60 \%$ of the companies had the higher level of disclosure. Singh (2009) studied 200 Indian companies and found 90\% of the companies had websites with $100 \%$ of them reporting any one of financial/investor relation/corporate information on their websites. Despina and Demetrios (2009) analyzed the internet reporting criteria followed by the 302 listed companies in Athens stock exchange and found all the companies had websites and reported some dimensions of financial information on their web pages. Further, result depicts that on average financial information (78.6\%) was highly disseminated on the web pages, followed by corporate governance information (53.3\%) and investor relation information $(49.7 \%)$. Pertiwi and Hermana (2013) compared the financial reporting on the internet between 25 Indonesian banks and nine non-banks and found the statistically significant difference in the internet financial reporting practices between the banks and non-banks in Indonesia. Alali 
and Romero (2012) investigated the use of corporate internet reporting by 84 publicly traded Argentina companies and reported $85.7 \%$ companies had websites and disseminating financial as well as nonfinancial information on their websites.

Thus, most of the studies were conducted by taking one dimension, i.e., content which included financial and non-financial information. Advance technology leads internet reporting to the utilization of two dimensions of disclosure, which are content and presentation (Khan and Ismail, 2012). Debreceny et al. (2002) studied the perceptions of the user regarding form and content, and it reveals that respondent ranked traditional financial highlights higher than dynamic attributes such as forwardlooking statement. Further, they found respondent preferred the new multimedia formats and navigation facilities such as audio/video, and hyperlink. Robbins and Stylianou (2003) investigated the content and design of global corporate websites. They found that content and presentation categories are significantly standardized among global corporations.

Kahn and Omar (2013) studied essential items of Internet financial reporting and they found financial items were necessary attributes of the content dimension. Multimedia and navigation were indispensable presentation attributes. Ilias et al., (2015) discovered an important presentation and content aspects of Internet business reporting among 212 Malaysia public listed companies. He added usability, accessibility, navigation, and timeliness are essential attributes of presentation features. Furthermore, financial statement, quarterly report, financial highlights, stock information, corporate governance, management team, auditor information, corporate social responsibility, online trading, and marketing are essential content attributes of websites. Omran \& Ramdhony (2016) analyzed 34 listed Mauritius companies' websites regarding content and presentation factors. There results depict $82 \%$ of content attributes, and $53 \%$ of presentation attributes were disclosed by Mauritius companies.
Further, regression results indicate that company size, board size, and liquidity were significant explanatory factors of internet financial reporting. Based on the literature review it becomes evident that content and presentations are the two fundamental dimensions of internet reporting. However, these two decisive factors are missing in Indian context see Garg and Verma (2010) and Singh (2009). Hence, the present study attempt to measure the extent of content and presentation features across the different sector of Indian companies.

Signaling theory also clarifies that organizations inside a similar industry tend to embrace a similar level of disclosure. If a company within industry neglects to take the same disclosure level, including web-based disclosure, like others in the similar industry at that point, it might be translated as a signal that the organization is concealing terrible news (Carven and Marston, 1999). There are numerous numbers of studies that analyze the relationship between the industry classification and the web-based reporting. The results for industry classification and internet reporting reported the mixed results likewise, Oyelere et al. (2003), Marston (2003), Xiao et al. (2004), Aly et al. (2010), Uyar (2011), Kamel and Awadallah (2017), found a significant association between industry classification and web-based corporate reporting. On the contrary, Ginner (1997), Carven and Marston (1999), Prabowo and Angkoso (2007), Abdelsalam and Street (2007), found no relationship between industrial classification and internet reporting. Hence the disclosure literature shows the mixed results between the corporate web disclosure and the industry classification. Thus the following hypothesis is purposed for the study:

H0: There is no significant difference in the industry type and the corporate web reporting.

The alternate hypothesis for the purposed hypothesis is:

H1: There is a significant difference in the industry type and the corporate web reporting. 


\section{RESEARCH METHODOLOGY}

The section describes the methodology used in the study including sample size, design and data collection.

\section{Sample}

183 companies based on market capitalization listed on the BSE are selected from the BT-1000 database for the year 2015-16 for this study. The selected companies are categorized broadly into sixteen sectors to measure the extent of internet reporting across different sectors. The sampled companies are given in Appendix.

\section{Data Collection}

The study is based on secondary data and the first step in the data collection is to identify the websites of the respective company. Thus, to locate the website of the company leading search engines such as Google (www.google.com) has been used. Thereafter, information on the web was measured with the help of internet disclosure index (IDI). The data was collected from the websites of sample companies during October-November, 2016.

\section{Scoring Scheme}

The internet disclosure index (IDI) is prepared to identify whether companies are utilizing the internet technology for reporting or not. The IDI is prepared from previous research (Robbins and Stylianou, 2003; Debreceny et al. 2002, Khan and Omar, 2013) and is modified as per the requirement of study and also satisfy the Indian context. The score of 1 is awarded if the attribute from the IDI is presented on the webpage, otherwise 0 is assigned (Lymer, 2000; Debreceny et al., 2002; Garg and Verma, 2010; Singh, 2009). The formula for calculating total score is as follow:

IDIS $=$ Sri

$\mathrm{i}=1$
Where, IDIS is Internet Disclosure Index Score ri is 1 if attribute found otherwise 0

$\mathrm{i}$ is $1,2,3,4 \ldots \ldots . .122$.

The IDI consist 122 attributes and these attributes have been broadly categorized into two groups, namely content (6 attributes) and presentation (4 attributes). Content dimension have been further sub-categorized into six groups, namely financial information (31 attributes), company information (15 attributes), stakeholder support (8 attributes), Human resources and employment opportunity ( 9 attributes), social responsibility (8 attributes), and marketing information (9 attributes). The presentation dimension has been subcategorized into four groups, namely, multimedia (5 attributes), navigation (13 attributes), security (6 attributes), and user support (18 attributes).

\section{FINDINGS AND DISCUSSION}

The section represents the results and analysis relating to corporate web reporting across the different industry groups. Of the 183 companies, 99 percent companies have active websites and disseminating financial and non-financial information on their web pages. Analysis Of Variance (ANOVA) was conducted to examine the significant variations of the each of the content and presentation attributes between the classified industries groups (see Robbins and Stylianou, 2003). Industry categories were used as dependent variables, and the content and design attributes were used as independent variables. The next paragraph presents the analysis on two broad categories and their subcategories.

\section{Extent of Content Features across Different Industry Groups}

As indicated in Table 1, most of the financial attributes has been disclosed by the majority of companies across the different sectors. The ANOVA value for historical share prices, share performances share day high/low and monthly high/low significantly different across sectors. The significant 
difference is observed in the disclosure of analyst report, the number of analyst following the firm, and analyst contact details. Disclosure of press releases and information on subsidiaries has the significant difference at 5 percent level of significance. All other financial attributes are not significantly different across the industry groups.

Most of the companies complied with the required disclosure of corporate governance information The ANOVA value for corporate governance category indicate that governance attributes does not significantly different at 5 percent level of significance except the attribute business risk analysis.

The higher variation is observed in the disclosure of corporate social responsibility across the different industry groups. Six out of nine attributes of the corporate social responsibility section have significant differences at one percent level of significance. The attributes which are significantly different are environmental spending \& activities, corporate social responsibility policy, social community engagement, sustainability report, and innovation and expansion.

In the human resources and employment opportunities, the result indicates that the attributes feature stories \& interviews of the employee, employee welfare \& perquisites, employee health \& safety, and employment results are significantly different across the different industry groups.

The result further shows that all the marketing information related attributes significantly different at 5 percent level of significance expect the disclosure of product and services attribute. Lastly, in the stakeholder support attributes disclosure of postal address of the company, company email-id, and frequently asked questions are significant at 5 percent level of significance.

\section{Extent of Presentation Features across Different Industry Groups}

The Table 2 depicts that there is standardization in the utilization of multimedia attributes across the different industry groups as attributes frames, flashes, graphics and interactive chart groups are not significantly different. However, the webcasting attribute is significantly different at 10 percent level of significance. Furthermore, the majority of navigation attributes are also standardized across the different industry groups, as the ANOVA values are less significant. 


\begin{tabular}{|c|c|c|c|c|c|c|c|c|c|c|c|c|c|c|c|c|}
\hline \multirow{2}{*}{ 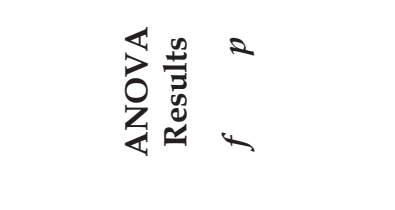 } & & तె & సิ & సิ & 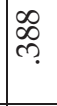 & 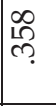 & 윰 & $\begin{array}{l}\infty \\
\infty \\
\infty\end{array}$ & ई. & f̊ & $\hat{0}$ & 苾. & 市 & m. & $\begin{array}{l}* \\
\tilde{O} \\
\tilde{\sigma} .\end{array}$ & ડ્ત \\
\hline & & 유. & 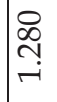 & $\begin{array}{l}\stackrel{\infty}{2} \\
\text { N̦ } \\
-\end{array}$ & $\underset{\text { iे }}{0}$ & I & $\widetilde{\Omega}$ & के & $\stackrel{N}{N}$ & $\stackrel{\infty}{q}$ & స్త్ & $\mid \begin{array}{l}9 \\
\infty \\
\infty \\
- \\
\end{array}$ & N̂. & 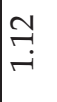 & 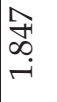 & $\begin{array}{l}\text { స్రి } \\
\text { - }\end{array}$ \\
\hline sәвехәмәg ج8 роо & & $\underset{-1}{\stackrel{1}{-1}}$ & $\underset{1}{8}$ & $\underset{-1}{8}$ & $\underset{1}{8}$ & $\underset{-1}{8}$ & $\underset{-1}{8}$ & 암 & 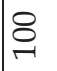 & $\underset{\ominus}{8}$ & 8 & 암 & $\infty$ & 암 & $\infty$ & 암 \\
\hline 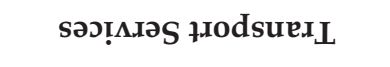 & & $\underset{\sim}{\stackrel{2}{2}}$ & $\underset{\sim}{\stackrel{1}{1}}$ & $\underset{\sim}{\stackrel{一}{2}}$ & $\underset{\sim}{\stackrel{9}{1}}$ & $\underset{\sim}{\stackrel{一}{(1)}}$ & $\underset{\sim}{\stackrel{0}{2}}$ & $\infty$ & 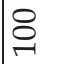 & 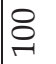 & ㅈ & 0 & $\underset{1}{8}$ & 8 & $\underset{1}{8}$ & ণิ \\
\hline uo!̣pnxұsuoว & & $\stackrel{2}{\circ}$ & $\stackrel{\circ}{\circ}$ & $\stackrel{一}{\circ}$ & $\stackrel{ }{0}$ & $\underset{\sim}{\stackrel{一}{0}}$ & $\stackrel{\ominus}{0}$ & $\infty$ & $\underset{\sim}{8}$ & 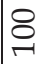 & 아 & $\infty$ & 움 & $\underset{8}{8}$ & 욱 & ঃิ \\
\hline [е!ฺиеu! хәчњО & & 20 & $\underset{1}{8}$ & $\underset{\sim}{\stackrel{2}{2}}$ & $\underset{\sim}{\stackrel{1}{2}}$ & ஜn & $\dddot{2}$ & 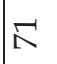 & L2 & ৪ & 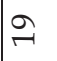 & $A$ & Lू & $\infty$ & $\infty$ & 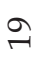 \\
\hline spoog & & ন & $\underset{1}{8}$ & $\underset{\sim}{8}$ & $\alpha$ & $\approx$ & $\underset{\sim}{\stackrel{2}{2}}$ & 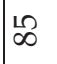 & $\underset{\sim}{\stackrel{一}{~}}$ & $\underset{\sim}{\stackrel{一}{2}}$ & \& & 苚 & $\alpha$ & 8 & $\infty 8$ & f \\
\hline sЈ!̣nәəеuxечd & & $\underset{-}{8}$ & $\underset{-1}{8}$ & $\underset{-}{8}$ & $\underset{-1}{8}$ & $\underset{\bullet}{8}$ & $\underset{-1}{\stackrel{-}{2}}$ & $\stackrel{\infty}{\infty}$ & $\underset{\sim}{8}$ & $\underset{\sim}{8}$ & $\bar{m}$ & m & $\underset{-1}{8}$ & o & $\infty$ & $\vec{m}$ \\
\hline Квобоичрағ & & $\stackrel{8}{\circ}$ & $\stackrel{1}{2}$ & $\stackrel{8}{\circ}$ & $\underset{-1}{8}$ & \& & $\underset{-1}{8}$ & ஜ & $\underset{\sim}{\stackrel{8}{0}}$ & $\stackrel{8}{\circ}$ & $\infty$ & $\infty$ & $\infty$ & 6 & $m$ & $\infty$ \\
\hline słonpoxd xəunsuo & & ガ & જ゙ & Ð゙ & హ゙ & $\infty$ & ガ & $\infty$ & ガ & ガ & ம & $\infty$ & A & $\infty$ & $\infty$ & $\infty$ \\
\hline 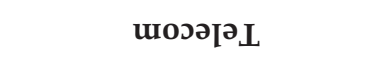 & & $\underset{-1}{8}$ & $\underset{-1}{8}$ & $\underset{7}{8}$ & $\underset{-1}{8}$ & 8 & $\underset{-1}{8}$ & $\infty$ & $\underset{\sim}{8}$ & $\underset{\sim}{8}$ & $m$ & $m$ & 8 & $\leadsto$ & $m$ & டி \\
\hline s[еग!̣uәчد & & $\stackrel{8}{\circ}$ & $\stackrel{8}{\circ}$ & $\stackrel{8}{\circ}$ & $\stackrel{8}{\circ}$ & $\stackrel{8}{\circ}$ & $\stackrel{8}{\circ}$ & டீ & $\stackrel{一}{\circ}$ & $\stackrel{一}{\circ}$ & 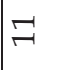 & $\exists$ & $\stackrel{8}{\circ}$ & i̊ & 6 & $\exists$ \\
\hline sə[!qourołn & & 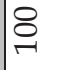 & $\stackrel{8}{-1}$ & \& & $\underset{-1}{8}$ & 8 & 8 & $\stackrel{\infty}{\wedge}$ & \& & 8 & ก & $m$ & 8 & $m$ & $\stackrel{8}{8}$ & ส \\
\hline 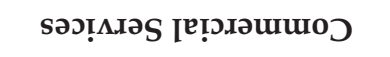 & & $\infty$ & $\infty$ & $\infty$ & $\infty$ & $\infty$ & $\infty$ & 8 & $\infty$ & $\infty$ & i & 0 & $\infty$ & i̊ & 아 & 암 \\
\hline sว!ฺ!!!ฺก & & $\underset{-}{8}$ & $\underset{-1}{8}$ & $\underset{-}{8}$ & $\underset{-1}{8}$ & 8 & ন & 尺 & $\underset{\sim}{8}$ & $\alpha$ & $m$ & $\stackrel{n}{\sim}$ & $\infty$ & \% & $\hat{N}$ & กิ \\
\hline su!yueg & & $\underset{-}{8}$ & $\underset{1}{8}$ & $\underset{-}{8}$ & $\underset{\sim}{\stackrel{2}{1}}$ & $\underset{\sim}{\stackrel{一}{ }}$ & $\underset{\sim}{\stackrel{2}{1}}$ & た & 命 & 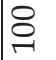 & $\stackrel{0}{\sim}$ & ন̀ & $\underset{\sim}{\&}$ & 迕 & 5 & 욱 \\
\hline [вæә & & न & ๙ & $\sigma$ & न & $\underset{\sim}{\stackrel{一}{2}}$ & 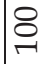 & $\infty$ & $\sigma$ & 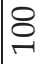 & œ & הิ & $\infty$ & m & $\infty$ & 过 \\
\hline seכ pue I!O & & $\underset{-1}{8}$ & $\underset{-1}{\varnothing}$ & $\underset{-1}{8}$ & $\underset{-1}{\varnothing}$ & $\underset{-1}{8}$ & $\underset{-1}{\stackrel{1}{2}}$ & $\curvearrowright$ & $\underset{\sim}{\circledR}$ & $\underset{\sim}{\circledR}$ & 아 & ஜิ & 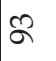 & in & $\mathbb{N}$ & $m$ \\
\hline 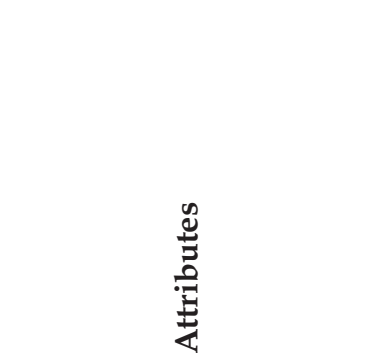 & 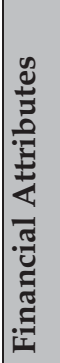 & 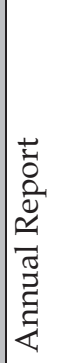 & 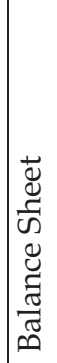 & 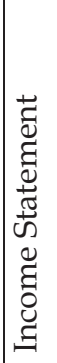 & $\begin{array}{l}3 \\
\frac{3}{11} \\
\frac{1}{11} \\
0 \\
0 \\
0\end{array}$ & 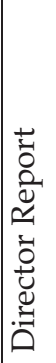 & 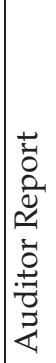 & 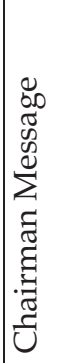 & 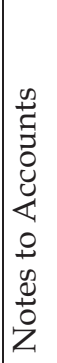 & 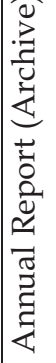 & 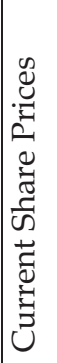 & 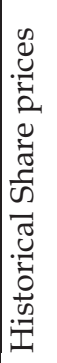 & 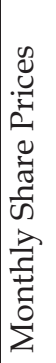 & 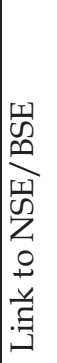 & 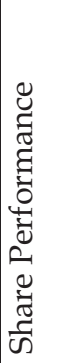 & 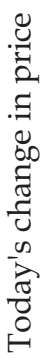 \\
\hline
\end{tabular}




\begin{tabular}{|c|c|c|c|c|c|c|c|c|c|c|c|c|c|c|c|c|c|c|}
\hline 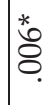 & 腒 & $\stackrel{*}{*}$ & 。ُ & $\stackrel{*}{*}$ & $\stackrel{r}{\partial}$ & ఫొ & $\nexists$ & 菼 & : & $\begin{array}{l}\text { 足 } \\
\infty\end{array}$ & 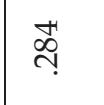 & $\stackrel{*}{*}$ & ث্ & $\overrightarrow{8}$ & 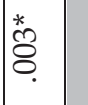 & $\begin{array}{l}\overrightarrow{5} \\
\overrightarrow{0} \\
\overrightarrow{0}\end{array}$ & 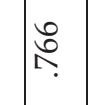 & 今ે \\
\hline i & $\mid \begin{array}{l}\infty \\
\stackrel{\leftrightarrow}{+}\end{array}$ & तี & $\begin{array}{l}\vec{F} \\
\vec{c}\end{array}$ & 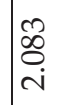 & $\begin{array}{l}\text { İ } \\
\text { ָे }\end{array}$ & ळু & $\begin{array}{l}\infty \\
\substack{n \\
n}\end{array}$ & 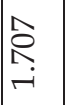 & 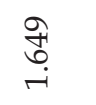 & స్ర & 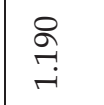 & 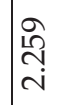 & ণ্ণ & 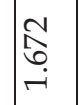 & $\underset{\mathcal{A}}{\mathcal{A}}$ & 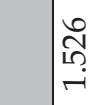 & $\stackrel{2}{2}$ & ్ֶָ \\
\hline 8 & \& & F & 0 & 0 & ते & 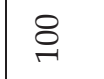 & $\infty$ & 0 & 0 & 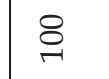 & fr & 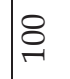 & fi & 8 & fr & 0 & 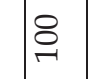 & $\infty$ \\
\hline$\sqrt{ }$ & iิ & 0 & 0 & 0 & 0 & 8 & $\underset{\sim}{\stackrel{一}{2}}$ & 8 & 0 & 8 & 0 & $\underset{\sim}{\stackrel{一}{2}}$ & 8 & $\stackrel{一}{\circ}$ & ì & 0 & $\infty$ & ते \\
\hline 아 & or & 0 & 0 & 0 & ণิ & $\infty$ & $\stackrel{ }{\circ}$ & 8 & 0 & $\infty$ & 8 & ஓ & $\infty$ & $\stackrel{\circ}{\circ}$ & $\infty$ & 0 & $\underset{\sim}{\stackrel{2}{2}}$ & 8 \\
\hline$\vec{r}$ & 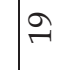 & 0 & in & œ & $\nexists$ & $\vec{\infty}$ & ฉू & $\infty$ & 0 & \& & $\infty$ & $\infty$ & กิ & 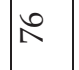 & in & 0 & 8 & $\infty$ \\
\hline$\infty_{\infty}^{\infty}$ & $\infty$ & $\infty$ & 0 & $\infty$ & L & 8) & $\bowtie$ & $\begin{array}{l}\infty \\
\infty \\
\infty\end{array}$ & $\infty$ & 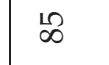 & 유 & 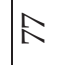 & $\infty$ & \& & 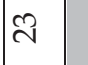 & $\stackrel{2}{\sim}$ & $\underset{\sim}{\stackrel{p}{ }}$ & H' \\
\hline$\sqrt{3}$ & $\stackrel{2}{\rightarrow}$ & $\ddot{\sim}$ & $\ddot{\sim}$ & $\stackrel{2}{\sim}$ & $\mathscr{f}$ & $\alpha$ & $\alpha$ & $\widehat{\sigma}$ & $\infty$ & $\alpha$ & $\stackrel{\sim}{\sim}$ & $\stackrel{一}{\circ}$ & 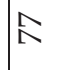 & $\infty$ & $\widehat{\sigma}$ & $\infty$ & $\sigma$ & ガ \\
\hline 6 & $\hat{0}$ & 0 & 6 & in & in & $\stackrel{9}{\circ}$ & 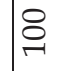 & 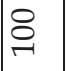 & 0 & $\infty$ & 0 & $\underset{\sim}{\stackrel{一}{2}}$ & in & $\hat{0}$ & $\Rightarrow$ & 0 & $\infty$ & in \\
\hline F & $F$ & $\stackrel{\rightarrow}{ }$ & 6 & 6 & $\infty$ & 8 & $\infty$ & in & 0 & $\infty$ & $\infty$ & J & $\mathbb{F}$ & $\stackrel{\circ}{\circ}$ & F & 6 & $\underset{\sim}{\stackrel{一}{ }}$ & in \\
\hline in & $m$ & 0 & $\Rightarrow$ & $\triangleq$ & $\therefore$ & $\infty$ & $\infty$ & $\hat{O}$ & $m$ & 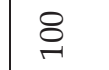 & 0 & $\underset{ }{\stackrel{2}{2}}$ & $\hat{\theta}$ & $\hat{0}$ & in & 0 & $\infty$ & in \\
\hline$F$ & $\exists$ & 0 & $\Rightarrow$ & 0 & 0 & ஜ̊ & $\underset{\sim}{\stackrel{一}{2}}$ & ஜ & 0 & $\stackrel{\infty}{\wedge}$ & 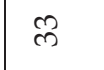 & $\hat{0}$ & $\hat{0}$ & $\propto$ & A & 0 & $\infty$ & $\tilde{\lambda}$ \\
\hline & $\approx$ & F & 0 & 0 & $\approx$ & $\stackrel{\infty}{\wedge}$ & $\infty$ & $\hat{0}$ & 0 & $\infty$ & $F$ & $\infty$ & ஜ & $\underset{ }{\stackrel{9}{2}}$ & $\hat{0}$ & 0 & $\underset{\sim}{\stackrel{ }{q}}$ & $\underset{ }{\stackrel{7}{~}}$ \\
\hline$q$ & নি & 0 & 0 & 0 & P & $\infty$ & 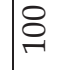 & 0 & 0 & $\infty$ & ฉิ & శิ & 8 & 8 & $\infty$ & g & $\underset{\sim}{\stackrel{一}{~}}$ & q \\
\hline 0 & 0 & $\infty$ & $\stackrel{2}{2}$ & 0 & $\infty$ & $\stackrel{\llcorner}{\infty}$ & $\underset{\sim}{\stackrel{一}{2}}$ & $\ddot{n}$ & 0 & $\stackrel{\sim}{\circ}$ & 나 & $\infty$ & \& & $\widehat{N}$ & to & $\stackrel{2}{\sim}$ & $\approx$ & $\widehat{్}$ \\
\hline 6 & 0 & $\stackrel{ }{\Rightarrow}$ & 0 & 0 & ले & 18 & ฉ & กิ & $\stackrel{\oplus}{\rightarrow}$ & \& & $\Rightarrow$ & ฉ & $\vec{\infty}$ & 5 & 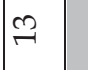 & $\stackrel{m}{\Rightarrow}$ & హ゙ & 通 \\
\hline ? & $\widehat{\curvearrowright}$ & a & 0 & 0 & $\stackrel{\infty}{\sim}$ & 员 & न & LI & 0 & 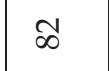 & તे & $\approx$ & $\Re$ & 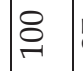 & À & ते & $\underset{\sim}{\stackrel{\sim}{~}}$ & Lf \\
\hline ते & $\ltimes$ & 0 & 0 & 0 & $\stackrel{\rho}{\rightarrow}$ & $\infty$ & 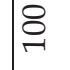 & 今 & 0 & $\infty$ & $\infty$ & $\infty$ & $\infty$ & $\Delta$ & กิ & 0 & $\underset{\sim}{\stackrel{一}{\prime}}$ & q \\
\hline 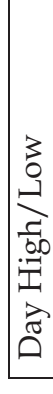 & 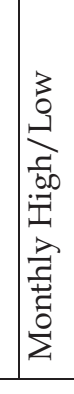 & 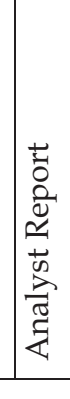 & 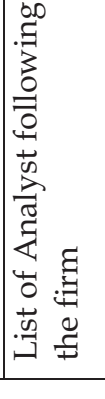 & 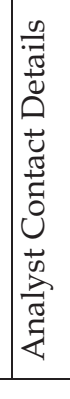 & 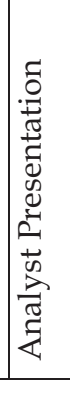 & 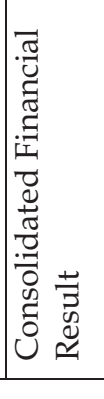 & 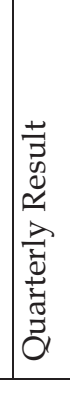 & 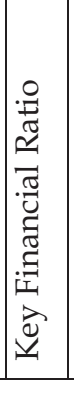 & 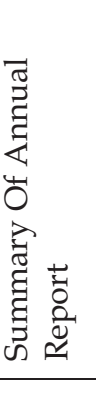 & 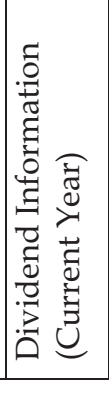 & 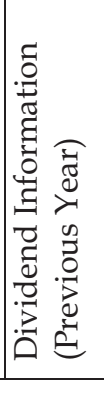 & 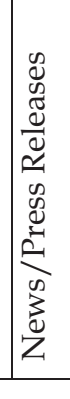 & 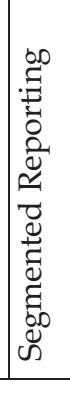 & 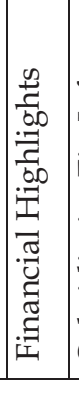 & 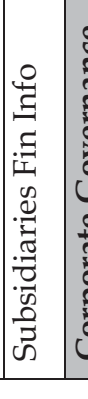 & 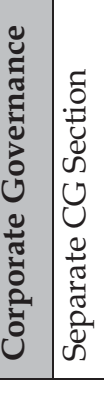 & 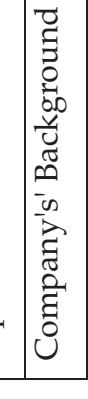 & 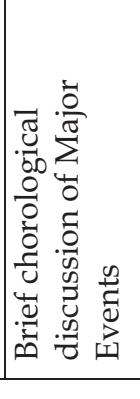 \\
\hline
\end{tabular}




\begin{tabular}{|c|c|c|c|c|c|c|c|c|c|c|c|c|c|c|c|c|c|}
\hline 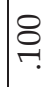 & 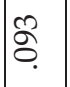 & 苍. & స్రి & 品 & \& & $\stackrel{*}{*}$ & $\stackrel{\infty}{\stackrel{+}{+}}$ & 冓 & $\stackrel{\infty}{=}$ & 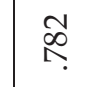 & $\stackrel{0}{\circ}$ & & $\stackrel{*}{\stackrel{*}{\circ}}$ & 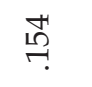 & 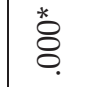 & 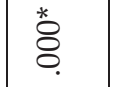 & ڤે \\
\hline & $\mid \begin{array}{l}\vec{n} \\
\text { hn } \\
\rightarrow-1\end{array}$ & مِ & $\begin{array}{l}\infty \\
\text { ò } \\
-i\end{array}$ & స్రీ & 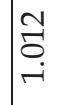 & $\begin{array}{l}\hat{O} \\
\stackrel{\mathrm{i}}{ }\end{array}$ & 암 & $\stackrel{\vec{\infty}}{\stackrel{\infty}{\infty} .}$ & 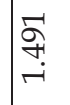 & 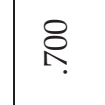 & $\begin{array}{l}\text { fि } \\
\text { ti }\end{array}$ & & $\mid \begin{array}{l}\vec{f} \\
\mathrm{i} \\
\mathrm{i}\end{array}$ & & 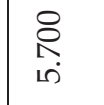 & $\begin{array}{c}\text { 员 } \\
\text { }\end{array}$ & 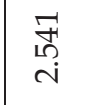 \\
\hline if & 0 & 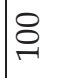 & 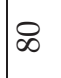 & 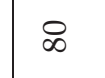 & 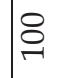 & ते & q & ir & 8 & $\stackrel{\leftrightarrow}{\circ}$ & 8 & & 8 & q & $\stackrel{\leftrightarrow}{\circ}$ & $\stackrel{一}{\circ}$ & $\triangleright$ \\
\hline 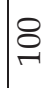 & F & 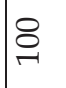 & $\stackrel{一}{\circ}$ & $\triangleright$ & $\infty$ & 0 & P & $\infty$ & $\stackrel{一}{\circ}$ & $\stackrel{8}{\circ}$ & 0 & & ন & o & o & $\triangleright$ & $\stackrel{ }{.}$ \\
\hline 8 & 0 & 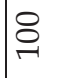 & 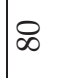 & $\infty$ & $\stackrel{\ominus}{\circledR}$ & $\infty$ & तి & $\infty$ & $\underset{\sim}{\stackrel{一}{2}}$ & $\infty$ & $\infty$ & & $\infty$ & $\underset{\sim}{\stackrel{7}{2}}$ & $\underset{7}{8}$ & 8 & $\underset{\sim}{8}$ \\
\hline in & 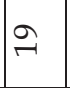 & 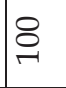 & $\vec{\infty}$ & $\stackrel{゚}{\curvearrowright}$ & 8 & $\stackrel{\infty}{\infty}$ & オ & in & $\infty$ & $\bowtie$ & $\widehat{\sigma}$ & & 9 & $\stackrel{\infty}{\infty}$ & ते & $\bowtie$ & ৪ \\
\hline 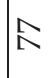 & $\infty$ & $\stackrel{\ominus}{\rightleftharpoons}$ & H' & bे & $\alpha$ & $\stackrel{\llcorner}{\sim}$ & $\vec{m}$ & \& & $\alpha$ & $\alpha$ & I & & $\widehat{\sigma}$ & $\mathscr{f}$ & $\hat{N}$ & 오 & ${ }_{\infty}^{10}$ \\
\hline 8 & 0 & $\underset{\sim}{\stackrel{一}{\prime}}$ & $\mathscr{\infty}$ & $\mathscr{L}$ & 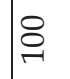 & $\infty$ & 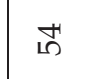 & के & $\underset{\sim}{\stackrel{一}{2}}$ & $\alpha$ & 으 & & $\widehat{\sigma}$ & $\vec{\omega}$ & $\infty$ & $\mathscr{\infty}$ & 8 \\
\hline$\infty$ & 0 & $\stackrel{\sim}{\circledR}$ & $\gtreqless$ & $\stackrel{\square}{~}$ & $\stackrel{一}{\circledR}$ & $\therefore$ & $\hat{\sigma}$ & in & $\hat{\theta}$ & $\infty$ & 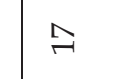 & & in & in & $\stackrel{9}{~}$ & $\infty$ & 8 \\
\hline ட̊n & $\stackrel{m}{\rightarrow}$ & tr & $\bar{\infty}$ & 8 & $\infty$ & $\stackrel{\Perp}{\mathrm{N}}$ & 8 & œ̊ & H & $\bar{\infty}$ & 8 & & 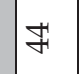 & in & 8 & $\vec{\infty}$ & ガ \\
\hline$\infty$ & 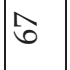 & $\underset{\sim}{\stackrel{一}{-1}}$ & $\infty$ & 6 & $\underset{\sim}{\stackrel{8}{2}}$ & $\ddot{m}$ & $\ddot{m}$ & 6 & $\underset{-1}{\stackrel{0}{2}}$ & $\infty$ & $\infty$ & & 0 & 6 & $\infty$ & B & 6 \\
\hline F & $F$ & 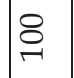 & فे & $\stackrel{\infty}{\wedge}$ & 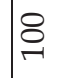 & ส & $\stackrel{\circ}{\circ}$ & ป & $\infty$ & $\infty$ & ส & & $\hat{\theta}$ & 0 & $\hat{\sigma}$ & $\infty$ & \& \\
\hline டొ & ส & $\stackrel{\ominus}{\ominus}$ & $\stackrel{\circ}{\llcorner}$ & $\stackrel{\infty}{\wedge}$ & $\infty$ & $\infty$ & $\exists$ & F & 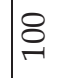 & $\infty$ & ف & & $F$ & $F$ & $\hat{\sigma}$ & $\hat{\sigma}$ & \\
\hline$\stackrel{\sigma}{-1}$ & or & $\stackrel{\oslash}{\circledR}$ & q & 8 & 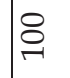 & 0 & q & 8 & 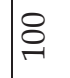 & $\triangleright$ & 8 & & F & or & 8 & $\triangleright$ & 8 \\
\hline స & $\vec{\infty}$ & 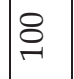 & $\infty$ & $\bowtie$ & 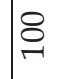 & $\infty$ & \& & dర & $\hat{A}$ & $\stackrel{\leftrightarrow}{\circ}$ & $\hat{N}$ & & $\mathscr{f}$ & f & $\hat{A}$ & $\alpha$ & \\
\hline$\widetilde{\sigma}$ & $\because$ & 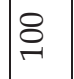 & ळ & 8 & 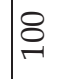 & $\Sigma$ & $\ddot{\lambda}$ & \& & $\infty$ & $\vec{\infty}$ & 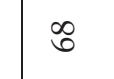 & & $\infty$ & ભે & $\ddot{\lambda}$ & ते & \\
\hline $\bar{\lambda}$ & 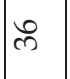 & 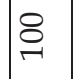 & $\cong$ & $\sigma$ & 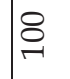 & ते & ते & ळ & 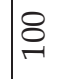 & 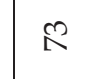 & تै & & in & பु & $\stackrel{9}{\circ}$ & $\infty$ & \\
\hline ర్ & ते & 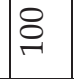 & N & $\infty$ & 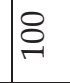 & 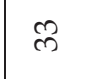 & q & q & $\underset{\sim}{\stackrel{一}{-1}}$ & $\underset{-}{\stackrel{9}{2}}$ & in & & 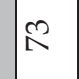 & in & 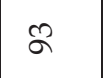 & 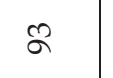 & \\
\hline$\frac{l_{0}}{.0}$ & 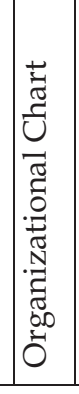 & 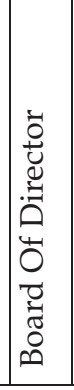 & 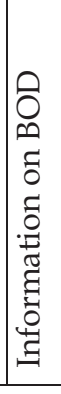 & 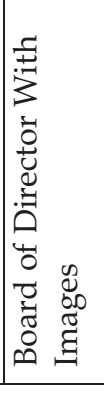 & 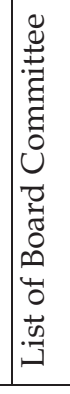 & 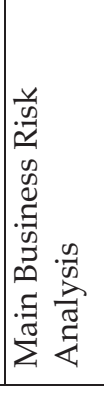 & 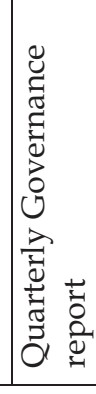 & 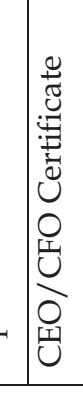 & 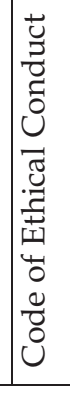 & 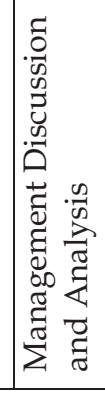 & 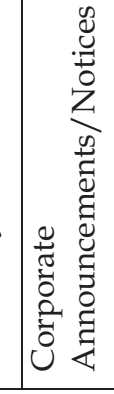 & 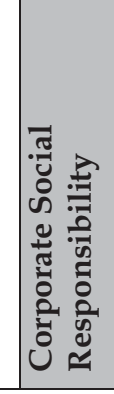 & 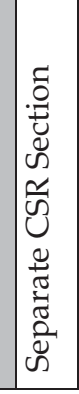 & 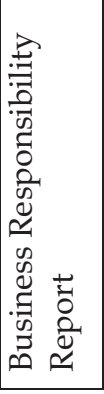 & 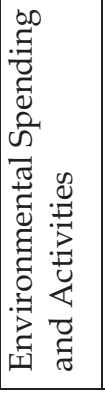 & 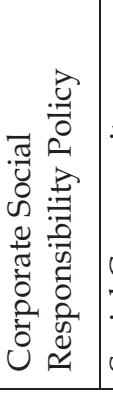 & 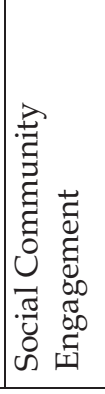 \\
\hline
\end{tabular}




\begin{tabular}{|c|c|c|c|c|c|c|c|c|c|c|c|c|c|c|c|c|c|}
\hline & \&̊ & 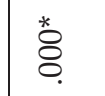 & & 各 & $\vec{\digamma}$ & స్ & $\begin{array}{l}* \\
\stackrel{*}{0} \\
\varnothing\end{array}$ & 范 & $\stackrel{*}{\circ}$ & $\stackrel{\rho}{\rho}$ & ठ́. & 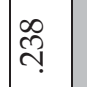 & & $\begin{array}{l}\text { ठ艹 } \\
\infty\end{array}$ & 荌 & \begin{tabular}{l}
$*$ \\
\hdashline \\
\hdashline
\end{tabular} & 茖 \\
\hline & ڤั & $\begin{array}{l}\stackrel{\circ}{ } \\
\text { id }\end{array}$ & & ఫু & 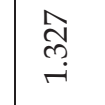 & & $\underset{\substack{\infty \\
\stackrel{\infty}{\infty}}}{ }$ & $\underset{\sim}{\stackrel{\sim}{-}}$ & $\begin{array}{l}\overrightarrow{\hat{i}} \\
\text { in }\end{array}$ & 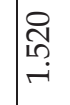 & $\begin{array}{l}\overrightarrow{0} \\
\stackrel{0}{+} \\
+\end{array}$ & స్తి & & 命 & $\begin{array}{l}\overrightarrow{5} \\
\overrightarrow{0} \\
\overrightarrow{0}\end{array}$ & $\begin{array}{l}\infty \\
\stackrel{\infty}{\sim} \\
i\end{array}$ & 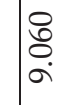 \\
\hline & 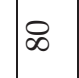 & 0 & & $\stackrel{\square}{\circ}$ & $\infty$ & 0 & o & 0 & 0 & Pr & 0 & $\infty$ & & $\stackrel{8}{\circ}$ & 0 & 0 & i \\
\hline & 8 & 0 & & 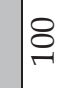 & 8 & of & of & ণి & of & 8 & 8 & 8 & & 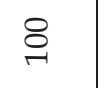 & নి & নి & $\infty$ \\
\hline & $\infty$ & 0 & & 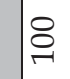 & 8 & i & 8 & of & of & P & 0 & గి & & $\underset{\sim}{\stackrel{8}{7}}$ & तి & P & 0 \\
\hline & $\checkmark$ & 0 & & $\lesssim$ & $\Sigma$ & 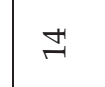 & $\stackrel{و}{\vec{r}}$ & in & in & in & 2 & $\infty$ & & $\stackrel{\square}{~}$ & $\stackrel{ }{\circ}$ & $\approx$ & $\infty$ \\
\hline & 8 & $\stackrel{\infty}{\infty}$ & & $\mathscr{\infty}$ & $\widehat{\sigma}$ & $\widetilde{\sigma}$ & $\vec{\infty}$ & $\stackrel{\infty}{\infty}$ & $\stackrel{\infty}{\infty}$ & $\AA$ & $\vec{m}$ & $\infty$ & & $\underset{\sim}{\stackrel{9}{9}}$ & 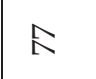 & 8 & $\infty$ \\
\hline & $\alpha$ & $\ddot{\gamma}$ & & $\alpha$ & $\stackrel{\infty}{\infty}$ & $\vec{\omega}$ & $\stackrel{\stackrel{2}{2}}{\sim}$ & 0 & \& & สู & 0 & S & & $\underset{\sim}{\stackrel{?}{2}}$ & $\bowtie$ & \& & $\infty$ \\
\hline & $\infty$ & $\widehat{\sigma}$ & & 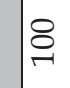 & $\widehat{\sigma}$ & in & $\widehat{B}$ & $m$ & $\infty$ & $\stackrel{8}{\circ}$ & 0 & $\infty$ & & $\underset{\sim}{\stackrel{丶}{二}}$ & 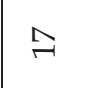 & 0 & 0 \\
\hline & $\vec{\infty}$ & $\mathbb{F}$ & & $\infty$ & in & $\underset{\infty}{\infty}$ & $\infty$ & $\stackrel{\text { L }}{i}$ & $\vec{m}$ & in & 0 & in & & హ゙ & $\stackrel{\infty}{\infty}$ & $\vec{m}$ & $\stackrel{2}{\sim}$ \\
\hline & in & $\therefore$ & & 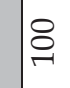 & $m$ & $m$ & in & in & $\Rightarrow$ & ¿ & 0 & $\infty$ & & $\underset{\sim}{\stackrel{8}{1}}$ & $\therefore$ & $A$ & in \\
\hline & $\infty$ & $m$ & & 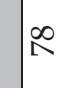 & F & F & ה & $m$ & ஜ & œீ & $F$ & m & & $\stackrel{?}{\circledR}$ & $\widehat{\sigma}$ & $F$ & ส \\
\hline & $\infty$ & ส & & $\infty$ & $\forall$ & ה & $\exists$ & c & ה & $m$ & 0 & F & & $\underset{\sim}{\stackrel{2}{2}}$ & F & $\infty$ & 0 \\
\hline & 8 & 0 & & 8 & 8 & ণి & ๙ి & or & q & 8 & f & o & & $\underset{\sim}{\stackrel{8}{2}}$ & 0 & $\stackrel{\sim}{.}$ & సি \\
\hline & $\lesssim$ & $\stackrel{\llcorner}{\sim}$ & & 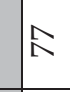 & H' & H' & $\infty$ & $\underset{c}{\infty}$ & $\infty$ & d & $\vec{\infty}$ & f & & 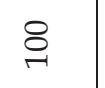 & $\infty$ & $\infty$ & $\infty$ \\
\hline & $I^{\prime}$ & $\infty$ & & $\vec{\infty}$ & $\infty$ & $\stackrel{\infty}{+}$ & 6 & ते & 우 & \$ా & 5 & $\stackrel{m}{2}$ & & $\underset{\sim}{\stackrel{9}{2}}$ & 0 & 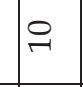 & 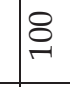 \\
\hline & $\sigma$ & $a$ & & $\sigma$ & 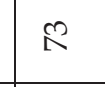 & $\stackrel{8}{F}$ & $\widehat{\lambda}$ & 另 & $\stackrel{\infty}{\sim}$ & 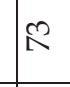 & $\stackrel{9}{\not}$ & $\infty$ & & $\stackrel{\sim}{\circ}$ & ळ & ते & N \\
\hline & $\Omega$ & $\stackrel{2}{\sim}$ & & 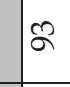 & $\hat{\theta}$ & in & $\stackrel{2}{\sim}$ & f & 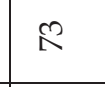 & $\infty$ & \& & of & & $\underset{\sim}{\stackrel{ }{2}}$ & 8 & $m$ & Aે \\
\hline & 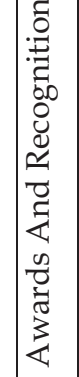 & 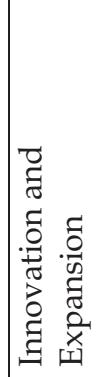 & 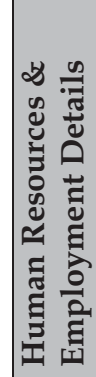 & 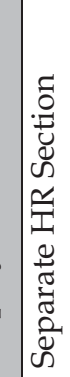 & 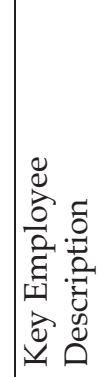 & 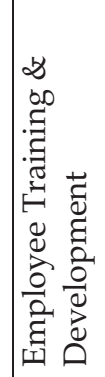 & 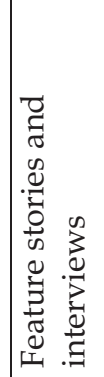 & 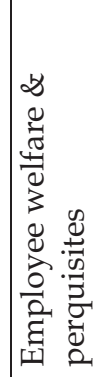 & 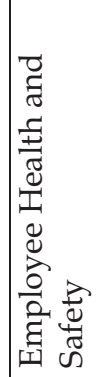 & 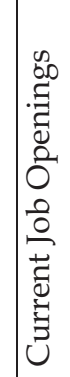 & 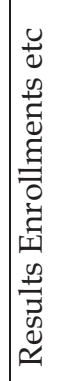 & . & 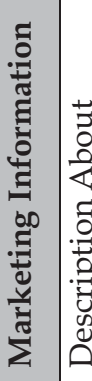 & 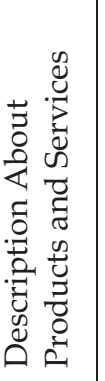 & 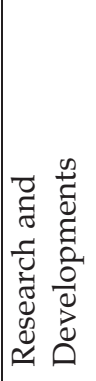 & 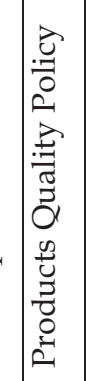 & 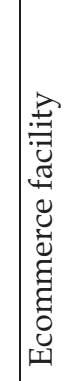 \\
\hline
\end{tabular}




\begin{tabular}{|c|c|c|c|c|c|c|c|c|c|c|c|c|c|}
\hline 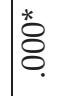 & 䓠 & $\stackrel{*}{8}$ & $\stackrel{*}{\circ}$ & 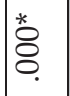 & & & $\underset{F}{F}$ & $\stackrel{\vec{\lambda}}{\text { ते }}$ & $\begin{array}{l}* \\
\stackrel{\infty}{0} \\
\stackrel{0}{0}\end{array}$ & 挡 & F. & 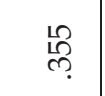 & $\stackrel{*}{*}$ \\
\hline$\underset{\sim}{\vec{c}}$ & 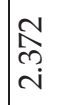 & 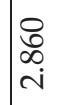 & $\begin{array}{l}\text { तु } \\
\text { ते }\end{array}$ & 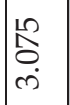 & & 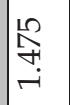 & 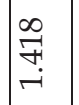 & $\begin{array}{c}\infty \\
\stackrel{\sim}{\sim} \\
\sim\end{array}$ & $\begin{array}{l}\stackrel{\infty}{\circ} \\
\stackrel{-}{r}\end{array}$ & $\mid \begin{array}{l}\infty \\
\infty \\
\infty \\
i\end{array}$ & $\underset{\vec{H}}{\overrightarrow{\mathcal{H}}}$ & $\underset{\rightarrow}{\stackrel{\leftrightarrow}{\rightarrow}}$ & 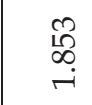 \\
\hline F & g & 0 & $\infty$ & f & & নे & $\infty$ & of & $\infty$ & $\infty$ & 8 & $\infty$ & సิ \\
\hline নি & g & 0 & 8 & 0 & & ते & 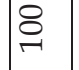 & of & q & 8 & 8 & $\infty$ & g \\
\hline 8 & i & 0 & 8 & 0 & & 0 & 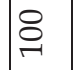 & 아 & $\stackrel{\sim}{\circ}$ & $\stackrel{\oslash}{\circledR}$ & 8 & $\infty$ & ণి \\
\hline in & is & 0 & $\widetilde{\sigma}$ & 0 & & $\exists$ & $\widehat{\sigma}$ & in & ㄴo & ஜ & กิ & $\widetilde{\sigma}$ & $\widetilde{\sigma}$ \\
\hline$\infty$ & \& & $\infty$ & 8 & 0 & & 苟 & $\infty$ & f & $\stackrel{\infty}{\infty}$ & 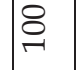 & $\AA$ & 8 & $\vec{m}$ \\
\hline 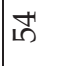 & 누 & 0 & f & 0 & & $\widetilde{N}$ & $\alpha$ & $\infty$ & 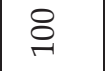 & $\alpha$ & $\infty$ & 8 & $\vec{\omega}$ \\
\hline$\infty$ & $\therefore$ & 0 & 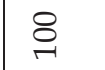 & 0 & & $m$ & $\stackrel{ }{\circ}$ & 0 & $\infty$ & $\Delta$ & in & $\underset{\sim}{\stackrel{0}{2}}$ & $\infty$ \\
\hline 8 & a & 0 & $\infty$ & 6 & & $\Rightarrow$ & $\infty$ & $\stackrel{2}{N}$ & $\underset{7}{8}$ & $\underset{\sim}{\stackrel{一}{-}}$ & $\infty$ & $\infty$ & $\stackrel{12}{1}$ \\
\hline in & $\hat{0}$ & 0 & $\hat{\sigma}$ & 0 & & 0 & $\infty$ & $\approx$ & $\hat{0}$ & $\hat{b}$ & 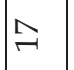 & $\infty$ & $\hat{\sigma}$ \\
\hline in & F & $\not{F}$ & $\stackrel{m}{m}$ & 0 & & $\infty$ & $\propto$ & $\infty$ & $\infty$ & $\infty$ & Z & 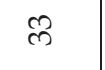 & $\not{+}$ \\
\hline$\stackrel{\infty}{\unrhd}$ & $\infty$ & $\not{F}$ & ळे & 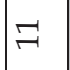 & & $\tilde{A}$ & $\hat{\sigma}$ & $\infty$ & $\stackrel{\infty}{\wedge}$ & $\infty$ & ட̊ & $\stackrel{\infty}{\wedge}$ & $\tilde{N}$ \\
\hline 8 & 아 & 0 & $\infty$ & 0 & & 8 & i & 8 & 8 & 8 & 아 & 8 & 8 \\
\hline$\stackrel{\sim}{\longrightarrow}$ & $\vec{m}$ & 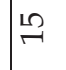 & แH & $\infty$ & & $\stackrel{2}{\sim}$ & 8 & $\stackrel{20}{\longrightarrow}$ & $\alpha$ & $\alpha$ & $\infty$ & $\infty$ & 苟 \\
\hline â & $\infty$ & $m$ & హో & $\stackrel{\oplus}{\rightarrow}$ & & $\because$ & 는 & $\stackrel{\vartheta}{ }$ & 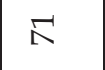 & $\vec{\sigma}$ & $\ddot{\lambda}$ & $\stackrel{10}{8}$ & $\infty$ \\
\hline$\stackrel{\curvearrowright}{\curvearrowright}$ & œ & $\widehat{\widehat{~}}$ & 字 & 0 & & $\infty$ & $\infty$ & లా & $\stackrel{一}{\circ}$ & 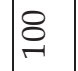 & If & న & $\hat{\curvearrowright}$ \\
\hline 8 & F & శి & q & $\stackrel{m}{\rightarrow}$ & & $\ltimes$ & 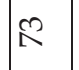 & q & $\propto$ & 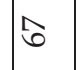 & f & $\triangleright$ & q \\
\hline 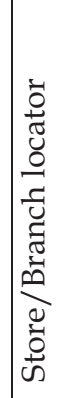 & 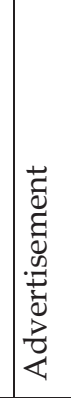 & 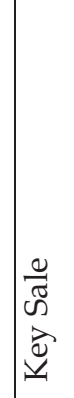 & 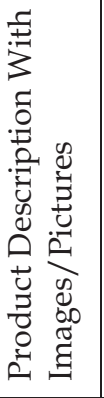 & 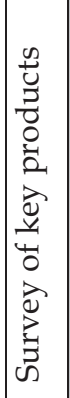 & 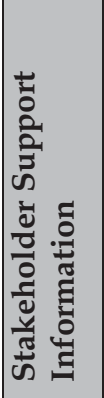 & 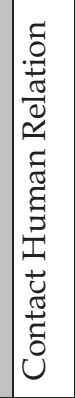 & 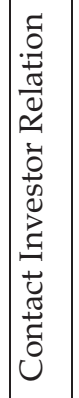 & 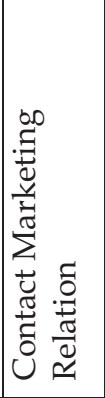 & 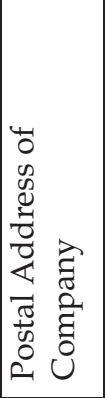 & 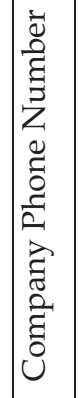 & 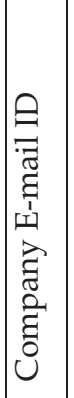 & 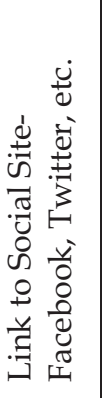 & 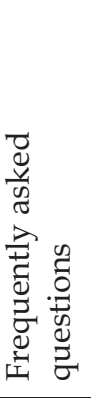 \\
\hline
\end{tabular}




\begin{tabular}{|c|c|c|c|c|c|c|c|c|c|c|c|c|c|c|}
\hline \multirow{2}{*}{ 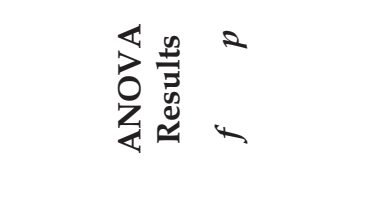 } & & ثै. & 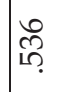 & $\tilde{\delta}$ & 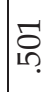 & 命 & & 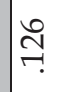 & 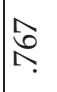 & 荅 & 艺 & $\begin{array}{l}* \\
\infty \\
0 \\
0\end{array}$ & ô. & $\begin{array}{l}0 \\
\text { 我 } \\
10\end{array}$ \\
\hline & & 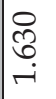 & $\widehat{\widehat{~}}$ & 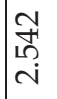 & 文 & 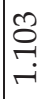 & & 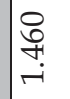 & $\stackrel{\operatorname{Ln}}{\stackrel{2}{\Gamma}}$ & $\begin{array}{l}\stackrel{0}{1} \\
\text { N }\end{array}$ & ळ̆ & 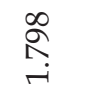 & 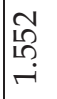 & $\stackrel{\infty}{\sigma}$ \\
\hline 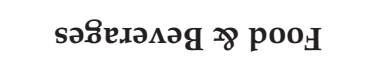 & & F & 8 & 8 & $\underset{-1}{8}$ & নి & & $\infty$ & $\underset{1}{8}$ & $\infty$ & $\stackrel{8}{\circ}$ & ஓ & $\underset{1}{8}$ & $\infty$ \\
\hline 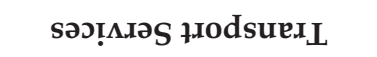 & & 아 & 8 & $\infty$ & 8 & 아 & & 8 & 8 & ণิ & $\infty$ & 8 & 8 & 8 \\
\hline uọ̣गn.ұsuoว & & 8 & 8 & ๙ิ & $\underset{1}{8}$ & নิ & & 8 & 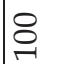 & ণิ & $\stackrel{-}{8}$ & 8 & 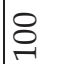 & $\stackrel{8}{8}$ \\
\hline [е!ฺиеч!н хәчłО & & กิ & $\infty$ & প & Lू & $\vartheta$ & & กิ & 8 & ৯े & $\underset{1}{8}$ & 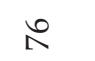 & $\stackrel{1}{\kappa}$ & $\infty$ \\
\hline spoog & & $\mathscr{q}$ & ช & 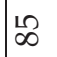 & 10 & $\vec{m}$ & & 6 & 8 & L & ส & $\alpha$ & $\underset{ }{8}$ & $\underset{7}{8}$ \\
\hline sว!̣nәэеuлечd & & மn & ชู & 6 & 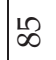 & $\ddot{N}$ & & 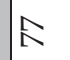 & $\underset{ }{8}$ & 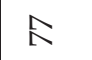 & $\stackrel{7}{2}$ & 익 & $\stackrel{2}{2}$ & $\stackrel{8}{\circ}$ \\
\hline 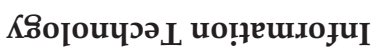 & & \& & $\infty$ & 8 & 8 & $m$ & & 8 & $\infty$ & 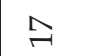 & 8 & $\infty$ & 8 & \& \\
\hline słonpoxd xəunsuoつ & & gิ & 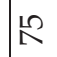 & $\infty$ & 8 & $\infty$ & & $\infty$ & ガ & ட & $\infty$ & $\vec{\infty}$ & $\stackrel{20}{2}$ & $\infty$ \\
\hline นоэәગə士 & & $m$ & $\hat{6}$ & $\infty$ & $\infty$ & $\approx$ & & 18 & $\infty$ & ○ & $\infty$ & $\infty$ & 8 & $\infty$ \\
\hline s[еग!̣uәчว & & ส & டீ & $\infty$ & $\infty$ & 0 & & $m$ & $\infty$ & டீ & 8 & $\hat{\theta}$ & 8 & $\underset{7}{8}$ \\
\hline so!!qouołn & & $\infty$ & 6 & $\infty$ & $\stackrel{8}{\circ}$ & ส & & டீ & $\infty$ & ส & $\infty$ & 8 & $\infty$ & $\underset{7}{8}$ \\
\hline 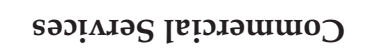 & & 아 & 8 & 8 & $\infty$ & 오 & & 8 & $\infty$ & ฉి & 8 & 아 & 8 & $\infty$ \\
\hline sว!ฺ!!!ฺก & & $\infty$ & 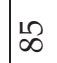 & ন & $\infty$ & $\infty$ & & 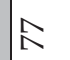 & $\alpha$ & $\infty$ & 8 & 8 & 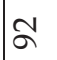 & 8 \\
\hline sulyueg & & নे & 8 & $\underset{7}{8}$ & $\underset{-1}{2}$ & 6 & & 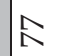 & 8 & ฉે & $\infty$ & 느 & $\infty$ & $\hat{~}$ \\
\hline [вұวW & & L & $\infty$ & $\infty$ & 웅 & $a$ & & 는 & $\sigma$ & ఈ゙ & \& & న & \& & \& \\
\hline seכ pue I!O & & $\hat{\theta}$ & $\sigma$ & $\underset{\sim}{\stackrel{2}{2}}$ & $\underset{ }{\stackrel{2}{2}}$ & $\ltimes$ & & $\infty$ & $\infty$ & $\hat{\theta}$ & 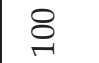 & $\infty$ & $\underset{\sim}{\stackrel{一}{2}}$ & $\infty$ \\
\hline 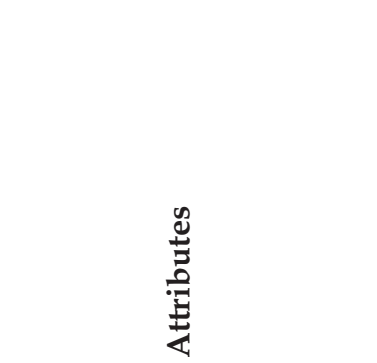 & 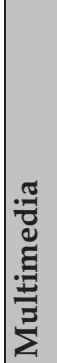 & 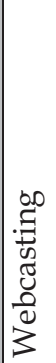 & 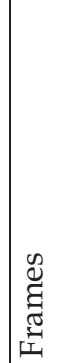 & 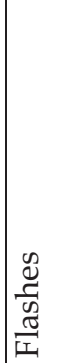 & $\begin{array}{l}0 \\
.0 \\
: \\
0 \\
0 \\
0 \\
0\end{array}$ & 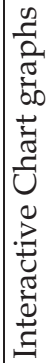 & 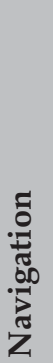 & 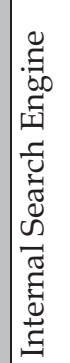 & 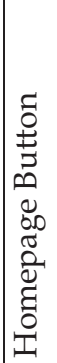 & 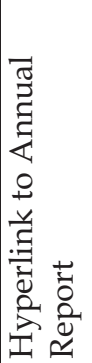 & 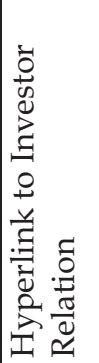 & 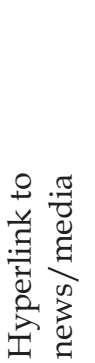 & 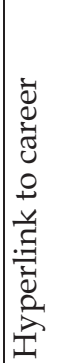 & 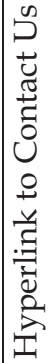 \\
\hline
\end{tabular}




\begin{tabular}{|c|c|c|c|c|c|c|c|c|c|c|c|c|c|c|c|c|c|c|c|c|c|}
\hline & ְ. & ণ্ & 疍 & $\stackrel{\mathscr{P}}{\stackrel{\leftrightarrow}{\varphi}}$ & $\begin{array}{l}\infty \\
1 \\
10\end{array}$ & 崩 & & గొ & 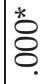 & 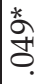 & $\stackrel{\mathscr{P}}{f}$ & 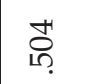 & & ठே. & 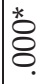 & \begin{tabular}{l}
$*$ \\
\multirow{2}{0}{} \\
$\stackrel{0}{0}$
\end{tabular} & & 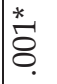 & $\stackrel{\stackrel{2}{7}}{\mathbb{7}}$ & 赵 & Әे \\
\hline 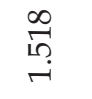 & $\stackrel{\curvearrowright}{\curvearrowright}$ & مَ & 苟 & $\stackrel{\overrightarrow{\tilde{D}}}{\stackrel{\sim}{r}}$ & $\begin{array}{l}\infty \\
\infty \\
\infty\end{array}$ & $\stackrel{\vec{H}}{\stackrel{\vec{f}}{r}}$ & & $\begin{array}{l}\text { స్ } \\
\infty\end{array}$ & $\begin{array}{l}\text { ते } \\
\text { ஸे }\end{array}$ & 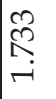 & 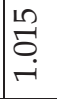 & ڤ̆ & & $\begin{array}{l}10 \\
\stackrel{1}{10} \\
-\end{array}$ & $\begin{array}{l}\infty \\
\infty \\
\infty \\
\dot{\infty}\end{array}$ & $\begin{array}{l}\stackrel{N}{N} \\
\stackrel{\sim}{\sim}\end{array}$ & & $\begin{array}{l}\overrightarrow{\mid} \\
\infty \\
\text { i }\end{array}$ & $\begin{array}{l}n \\
0 \\
0 \\
+ \\
+\end{array}$ & $\begin{array}{l}\Delta \\
\dot{\sim} \\
\stackrel{-}{n}\end{array}$ & m. \\
\hline 0 & 0 & $\infty$ & 8 & 0 & 0 & ㅇ & & 8 & ㄱ. & 0 & 요 & 0 & & ㅇ. & 요 & 0 & & 아 & 윽 & 우 & 0 \\
\hline 0 & 0 & $\underset{\sim}{\stackrel{2}{2}}$ & तิ & 0 & 0 & $\underset{\sim}{\stackrel{ }{2}}$ & & $\infty$ & 아 & 아 & 유 & 0 & & ิㅗ & 8 & $\stackrel{\sim}{ }$ & & 아 & $\underset{ }{\stackrel{8}{2}}$ & 아 & ন \\
\hline 0 & 0 & 유 & ㄱ. & 0 & 10 & $\infty$ & & $\triangleright$ & ฉి & 아 & 8 & 0 & & 0 & 0 & 0 & & ন & 8 & ชి & 0 \\
\hline 우 & 0 & $\infty$ & $\stackrel{\infty}{\infty}$ & 0 & 욱 & ช & & ஜ & $\bar{\infty}$ & $\stackrel{\infty}{+}$ & ন̀ & 욱 & & $\stackrel{2}{\rightleftharpoons}$ & ন̀ & $\stackrel{2}{7}$ & & $\infty$ & \& & $m$ & 으 \\
\hline$\infty$ & 0 & ช & $\ddot{N}$ & 0 & 0 & f & & $\stackrel{\square}{\infty}$ & for & f & $\approx \vec{~}$ & 0 & & $\vec{\infty}$ & Hn & $\ddot{N}$ & & f & $\underset{ }{\stackrel{-}{2}}$ & I & $\stackrel{12}{\longrightarrow}$ \\
\hline 0 & $\stackrel{2}{\sim}$ & 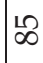 & เి & $\stackrel{\perp}{\sim}$ & $\infty$ & a & & $\underset{\sim}{\stackrel{一}{~}}$ & $\vec{\omega}$ & $\infty$ & $\stackrel{\perp}{\sim}$ & 0 & & $\infty$ & $\infty$ & 0 & & $\vec{m}$ & $\underset{-1}{8}$ & $\vec{m}$ & $\stackrel{10}{\longrightarrow}$ \\
\hline 오 & 0 & $\underset{\sim}{\stackrel{1}{2}}$ & $\stackrel{\curvearrowright}{\curvearrowright}$ & 0 & 0 & $\infty$ & & $\infty$ & 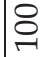 & in & 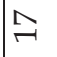 & $\widehat{ح}$ & & 0 & in & 0 & & $\hat{6}$ & $\underset{r}{8}$ & $\hat{\sigma}$ & $\curvearrowright$ \\
\hline$\stackrel{m}{-1}$ & $\stackrel{m}{\longrightarrow}$ & $\infty$ & $\infty$ & 6 & $\stackrel{\infty}{\rightarrow}$ & டீ & & $\stackrel{12}{1}$ & $\varpi$ & 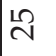 & $\stackrel{\infty}{\longrightarrow}$ & 0 & & 0 & $\stackrel{\infty}{\rightarrow}$ & 0 & & $\bar{\infty}$ & హ゙ & $\vec{\infty}$ & 6 \\
\hline$\widehat{ح}$ & 0 & $\infty$ & $\widehat{0}$ & 0 & $\stackrel{\wedge}{\sim}$ & $\hat{6}$ & & $\infty$ & $\infty$ & 0 & $\stackrel{ }{\sim}$ & 0 & & 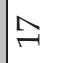 & $m$ & 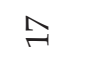 & & $\stackrel{\sim}{\sim}$ & $\underset{ }{\stackrel{8}{1}}$ & $\stackrel{\imath}{\neg}$ & $m$ \\
\hline 0 & $\exists$ & $\hat{\sigma}$ & 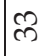 & 0 & 0 & $\not{F}$ & & $\stackrel{\infty}{1}$ & $\exists$ & $\approx$ & $\not{F}$ & 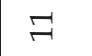 & & $\exists$ & $\ddot{m}$ & $F$ & & 0 & $\underset{-18}{8}$ & 0 & $\exists$ \\
\hline 0 & 0 & $\hat{\sigma}$ & ส & ন & 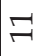 & டீ & & $\infty$ & $\infty$ & $\approx$ & $F$ & ส & & $\exists$ & 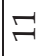 & 0 & & $m$ & $\infty$ & N & 0 \\
\hline 0 & 0 & $\underset{\sim}{\stackrel{2}{2}}$ & 아 & 0 & ㄱ. & $\infty$ & & $\underset{ }{\stackrel{ }{2}}$ & $\infty$ & 8 & 유 & 0 & & 아 & $\infty$ & 아 & & ㄱ. & 음 & 이 & శ \\
\hline$\stackrel{10}{\sim}$ & 0 & 10 & $\vec{m}$ & 0 & 0 & gి & & ন & in & $\stackrel{20}{\sim}$ & $\infty$ & $\infty$ & & $\infty$ & O & m & & I & 음 & lo & 0 \\
\hline$m$ & $m$ & ঃ & $\stackrel{L}{\infty}$ & $\infty$ & 0 & $\vec{\infty}$ & & $\infty$ & $\stackrel{10}{m}$ & $\ddot{\sim}$ & ले & 0 & & $\stackrel{m}{\rightarrow}$ & 估 & ले & & $\stackrel{\partial}{\rightleftharpoons}$ & 윽 & $\stackrel{2}{2}$ & 0 \\
\hline$a$ & $\sigma$ & | & $\stackrel{1}{f}$ & $a$ & $\stackrel{\infty}{\sim}$ & $\stackrel{N}{\wedge}$ & & 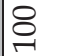 & 10 & 0 & 追 & 0 & & $a$ & $\stackrel{\leftrightarrow}{\not \rightarrow}$ & $\hat{\imath}$ & & ลิ & 욱 & ลे & $a$ \\
\hline$\stackrel{m}{\longrightarrow}$ & $\Lambda$ & $\Re$ & حิ & 0 & $\Lambda$ & $\hat{6}$ & & 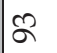 & 8 & $m$ & 요 & 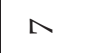 & & A & 8 & ลิ & & ลิ & 8 & ৯ิ & $\triangle$ \\
\hline 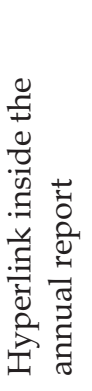 & 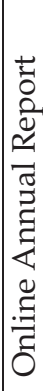 & 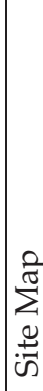 & 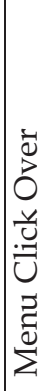 & 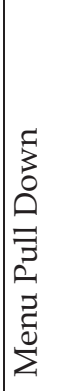 & 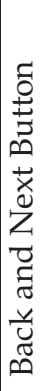 & 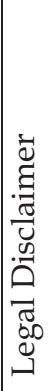 & 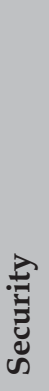 & 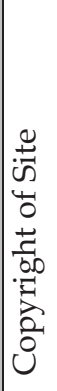 & 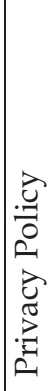 & 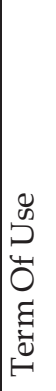 & 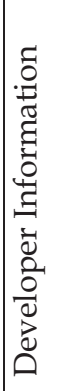 & 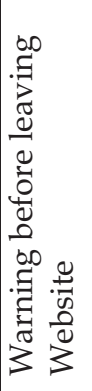 & 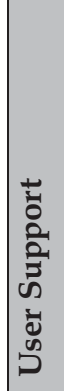 & 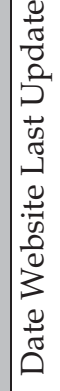 & 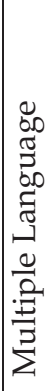 & 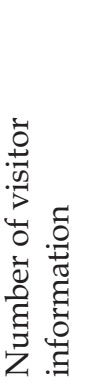 & 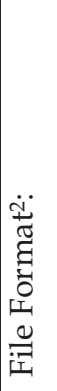 & 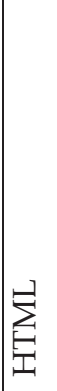 & 崖 & $\mid \overrightarrow{\underline{\hat{Q}}}$ & $\mid \begin{array}{c}\overrightarrow{\mathscr{U}} \\
x \\
x\end{array}$ \\
\hline
\end{tabular}




\begin{tabular}{|c|c|c|c|c|c|c|c|c|c|c|c|}
\hline ले & $\stackrel{\curvearrowright}{-}$ & 8 & సి & $\begin{array}{l}\stackrel{*}{\circ} \\
\stackrel{0}{0} .\end{array}$ & $\stackrel{*}{\circ}$ & * & 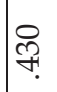 & 艾 & *ै & * & \\
\hline 苞 & مִ & $\begin{array}{l}\infty \\
\infty \\
\infty \\
\infty \\
\infty\end{array}$ & ثิ) & ले & $\begin{array}{l}\vec{N} \\
\stackrel{N}{N}\end{array}$ & $\begin{array}{l}\text { 扁 } \\
\text { iv }\end{array}$ & فે & 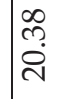 & $\begin{array}{l}R \\
\text { ભે } \\
\sigma\end{array}$ & 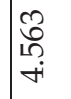 & \\
\hline 0 & 0 & 아 & 오 & 아 & 아 & 0 & 0 & $\underset{-}{8}$ & 0 & 오 & \\
\hline 0 & 0 & సి & ๙ & 8 & 0 & 0 & 아 & $\underset{\sim}{\stackrel{0}{2}}$ & 0 & 0 & \\
\hline 오 & 0 & 8 & 아 & 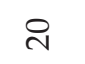 & 아 & 0 & 0 & $\underset{7}{8}$ & 암 & 0 & \\
\hline 0 & 0 & ถิ & $\nexists$ & $\widetilde{\sigma}$ & $\nexists$ & in & 0 & $\underset{-}{8}$ & $\stackrel{\vartheta}{\rightleftharpoons}$ & in & \\
\hline 0 & 0 & 8 & m & $\stackrel{\infty}{n}$ & $\bar{m}$ & 0 & 0 & $\underset{-1}{8}$ & $\infty$ & 0 & \\
\hline 0 & 0 & ம் & 0 & $\stackrel{\infty}{\infty}$ & 0 & $\infty$ & $\infty$ & $\underset{\sim}{8}$ & $\infty$ & 0 & \\
\hline 0 & 0 & $\hat{\sigma}$ & $\stackrel{\curvearrowright}{\sim}$ & 오 & $\widehat{\widehat{0}}$ & 0 & in & $\underset{\sim}{\stackrel{2}{2}}$ & $\stackrel{\sim}{\neg}$ & 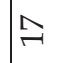 & \\
\hline 0 & 0 & டீ & 0 & $\not{F}$ & $\vec{m}$ & $\stackrel{M}{\longrightarrow}$ & 0 & హ゙ & $\stackrel{m}{\longrightarrow}$ & 6 & \\
\hline 0 & 0 & in & $\hat{A}$ & $\hat{\sigma}$ & $m$ & $\hat{}$ & $m$ & $\underset{1}{8}$ & $\ddot{m}$ & 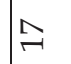 & \\
\hline 0 & 0 & $\infty$ & 0 & しొ & 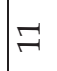 & 0 & 가 & $\infty$ & $\not$ & 0 & تُ \\
\hline$F$ & 0 & ட & $F$ & สี & $F$ & $F$ & 0 & $\infty$ & 0 & 0 & $\underset{\Xi}{\stackrel{E}{E}}$ \\
\hline 0 & 0 & 아 & 0 & 아 & 이 & 0 & 0 & $\underset{\sim}{\stackrel{9}{2}}$ & 0 & ते & $\begin{array}{l}\overrightarrow{0} \\
.00 \\
.0\end{array}$ \\
\hline 0 & 0 & $\bar{m}$ & $\bar{m}$ & ช్ & $\ddot{\lambda}$ & 0 & $\infty$ & $\infty 8$ & $\vec{\infty}$ & 0 & Z \\
\hline 0 & 0 & $\stackrel{\infty}{+}$ & নे & హ゙ & $\stackrel{0}{\sim}$ & $\stackrel{M}{\sim}$ & 6 & ล⿵ & 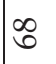 & $\circ$ & $\frac{\pi}{2}$ \\
\hline 0 & 0 & ठ゙ & 0 & in & $\stackrel{10}{7}$ & 0 & m & $\underset{-1}{8}$ & ลิ & 0 & $\stackrel{\text { O }}{\text { I }}$ \\
\hline 0 & 0 & 아 & 아 & 8 & 이 & 0 & 오 & $\underset{\sim}{\stackrel{2}{2}}$ & ลิ & $\ltimes$ & $\Xi$ \\
\hline 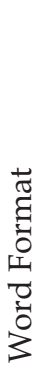 & 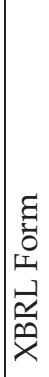 & 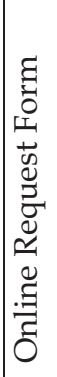 & 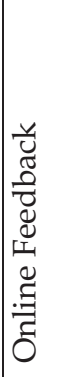 & 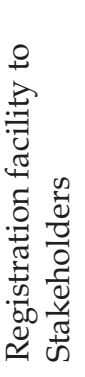 & 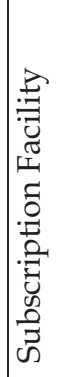 & 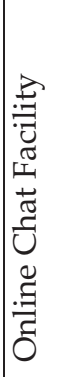 & $\begin{array}{l}\vec{D} \\
\mathbb{W} \\
0 \\
0 \\
0 \\
0\end{array}$ & 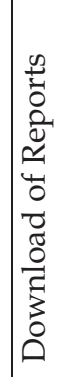 & 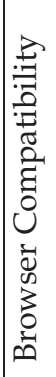 & 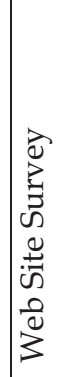 & 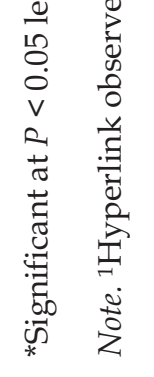 \\
\hline
\end{tabular}


The analysis of security attributes depicts that there are not such higher variations between the industry groups, only privacy policy and terms of use of website attribute significantly different across the industry groups.

In the vital attribute of presentations, user support (Table 2), we found that overall there is standardization in providing the user interface attributes. However, the significant difference is seen in the attributes multiple languages, the number of visitor information, financial information in HTML format, registration facility to stakeholders, email subscription facility of information, online chat facility, browser compatibility and website survey facility.

\section{Hypothesis Testing}

The analysis of extent of content and presentation features of website from Table 1 and 2 indicate that there are comparatively variations among the different industry groups. In order to confirm the variation in disclosure practices of different industry groups, analysis of variation test has been applied and the result of ANOVA is presented in Table 3. The results of ANOVA test reveals that there is a significant difference among the sixteen industries classification and corporate internet reporting at 5 percent of significance level $(\mathrm{F}=2.427, \mathrm{p}=0.03)$. Therefore, the null hypothesis has been rejected and the alternate hypothesis $\mathrm{H} 1$, which states that there are significant differences in the mean score of corporate web reporting and industry type, has been accepted. These results are consistent with previous research in different countries, which found an association between industry type and internet reporting. For example, Oyeler et al. (2003) reported an association between industry type and online reporting of New Zealand companies. Marston (2003) indicates that industry sector influences the disclosure practice of Japanese companies. Xiao et al. (2004) proved that corporate internet disclosure was greater among Chinese company in information technology sector.

Table 3 : ANOVA test for differences in Corporate Internet Reporting and Industry Groups

\begin{tabular}{|l|l|l|l|l|l|}
\hline & Sum of Squares & Df & Mean Square & F & p-value \\
\hline Between Groups & 4184.926 & 15 & 278.995 & 2.427 & $.003^{*}$ \\
\hline Within Groups & 19081.409 & 166 & 114.948 & & \\
\hline & 23266.335 & 181 & & & \\
\hline
\end{tabular}

*Note: The mean difference is significant at 0.05 levels.

Table 4 : ANOVA test Multiple Comparison - Scheffe

\begin{tabular}{|c|c|c|c|c|c|c|c|}
\hline \multirow{2}{*}{$\begin{array}{l}\text { Dependent } \\
\text { Variable }\end{array}$} & \multirow[t]{2}{*}{ Industry (I) } & \multirow[t]{2}{*}{ Industry (J) } & \multirow{2}{*}{$\begin{array}{l}\text { Mean } \\
\text { Diff } \\
(\mathrm{I}-\mathrm{J})\end{array}$} & \multirow[t]{2}{*}{ SE } & \multirow[t]{2}{*}{ Sig. } & \multicolumn{2}{|c|}{ 95\% Confidence Leve } \\
\hline & & & & & & $\begin{array}{l}\text { Lower } \\
\text { bound }\end{array}$ & $\begin{array}{l}\text { Upper } \\
\text { bound }\end{array}$ \\
\hline Total & Oil and Gas & $\begin{array}{l}\text { Metals and } \\
\text { Mining }\end{array}$ & 0.188 & 4.256 & 1.000 & -21.47 & 21.85 \\
\hline \multirow[t]{4}{*}{ Score } & & Banking & 4.572 & 3.372 & 1.000 & -12.59 & 21.74 \\
\hline & & Utilities & 1.503 & 4.063 & 1.000 & -19.18 & 22.18 \\
\hline & & $\begin{array}{l}\text { Commercial } \\
\text { Services }\end{array}$ & 10.533 & 5.537 & .999 & -17.65 & 38.71 \\
\hline & & Automobiles & 2.733 & 4.521 & 1.000 & -20.27 & 25.74 \\
\hline
\end{tabular}




\begin{tabular}{|c|c|c|c|c|c|c|}
\hline & Chemicals & 11.511 & 4.521 & .968 & -11.50 & 34.52 \\
\hline & Telecom & 4.567 & 5.179 & 1.000 & -21.79 & 30.93 \\
\hline & $\begin{array}{l}\text { Consumer } \\
\text { Products }\end{array}$ & -4.667 & 3.915 & 1.000 & -24.59 & 15.26 \\
\hline & IT & -11.433 & 5.179 & .992 & -37.79 & 14.93 \\
\hline & Pharmaceutics & 1.656 & 4.063 & 1.000 & -19.02 & 22.33 \\
\hline & Capital Goods & -1.421 & 4.063 & 1.000 & -22.10 & 19.26 \\
\hline & $\begin{array}{l}\text { Other Fin. } \\
\text { Services }\end{array}$ & 6.971 & 3.624 & .998 & -11.48 & 25.42 \\
\hline & Construction & -2.267 & 5.537 & 1.000 & -30.45 & 25.91 \\
\hline & $\begin{array}{l}\text { Transport } \\
\text { Services }\end{array}$ & 7.133 & 5.537 & 1.000 & -21.05 & 35.31 \\
\hline & $\begin{array}{l}\text { Food and } \\
\text { Beverages }\end{array}$ & 4.733 & 5.537 & 1.000 & -23.45 & 32.91 \\
\hline Metals and & Banking & 4.384 & 3.763 & 1.000 & -14.77 & 23.54 \\
\hline Mining & Utilities & 1.315 & 4.392 & 1.000 & -21.04 & 23.67 \\
\hline & $\begin{array}{l}\text { Commercial } \\
\text { Services }\end{array}$ & 10.345 & 5.783 & .999 & -19.09 & 39.78 \\
\hline & Automobiles & 2.545 & 4.819 & 1.000 & -21.98 & 27.07 \\
\hline & Chemicals & 11.323 & 4.819 & .985 & -13.20 & 35.85 \\
\hline & Telecom & 4.379 & 5.441 & 1.000 & -23.32 & 32.07 \\
\hline & $\begin{array}{l}\text { Consumer } \\
\text { Products }\end{array}$ & -4.855 & 4.256 & 1.000 & -26.52 & 16.81 \\
\hline & IT & -11.621 & 5.441 & .995 & -39.32 & 16.07 \\
\hline & Pharmaceutics & 1.469 & 4.392 & 1.000 & -20.89 & 23.82 \\
\hline & Capital Goods & -1.608 & 4.392 & 1.000 & -23.96 & 20.75 \\
\hline & $\begin{array}{l}\text { Other Fin. } \\
\text { Services }\end{array}$ & 6.784 & 3.990 & 1.000 & -13.53 & 27.09 \\
\hline & Construction & -2.455 & 5.783 & 1.000 & -31.89 & 26.98 \\
\hline & $\begin{array}{l}\text { Transport } \\
\text { Services }\end{array}$ & 6.945 & 5.783 & 1.000 & -22.49 & 36.38 \\
\hline & $\begin{array}{l}\text { Food and } \\
\text { Beverages }\end{array}$ & 4.545 & 5.783 & 1.000 & -24.89 & 33.98 \\
\hline Banking & Utilities & -3.069 & 3.543 & 1.000 & -21.10 & 14.96 \\
\hline & $\begin{array}{l}\text { Commercial } \\
\text { Services }\end{array}$ & 5.961 & 5.167 & 1.000 & -20.34 & 32.26 \\
\hline & Automobiles & -1.839 & 4.060 & 1.000 & -22.50 & 18.82 \\
\hline & Chemicals & 6.939 & 4.060 & 1.000 & -13.72 & 27.60 \\
\hline & Telecom & -0.005 & 4.782 & 1.000 & -24.34 & 24.33 \\
\hline & $\begin{array}{l}\text { Consumer } \\
\text { Products }\end{array}$ & -9.239 & 3.372 & .938 & -26.40 & 7.92 \\
\hline & IT & -16.005 & 4.782 & .734 & -40.34 & 8.33 \\
\hline & Pharmaceutics & -2.916 & 3.543 & 1.000 & -20.95 & 15.12 \\
\hline & Capital Goods & -5.993 & 3.543 & 1.000 & -24.02 & 12.04 \\
\hline & $\begin{array}{l}\text { Other Fin. } \\
\text { Services }\end{array}$ & 2.399 & 3.030 & 1.000 & -13.02 & 17.82 \\
\hline & Construction & -6.839 & 5.167 & 1.000 & -33.14 & 19.46 \\
\hline & $\begin{array}{l}\text { Transport } \\
\text { Services }\end{array}$ & 2.561 & 5.167 & 1.000 & -23.74 & 28.86 \\
\hline & $\begin{array}{l}\text { Food and } \\
\text { Beverages }\end{array}$ & 0.161 & 5.167 & 1.000 & -26.14 & 26.46 \\
\hline
\end{tabular}




\begin{tabular}{|c|c|c|c|c|c|c|}
\hline \multirow[t]{12}{*}{ Utilities } & $\begin{array}{l}\text { Commercial } \\
\text { Services }\end{array}$ & 9.031 & 5.642 & 1.000 & -19.69 & 37.75 \\
\hline & Automobiles & 1.231 & 4.649 & 1.000 & -22.43 & 24.89 \\
\hline & Chemicals & 10.009 & 4.649 & .994 & -13.65 & 33.67 \\
\hline & Telecom & 3.064 & 5.292 & 1.000 & -23.87 & 30.00 \\
\hline & $\begin{array}{l}\text { Consumer } \\
\text { Products }\end{array}$ & -6.169 & 4.063 & 1.000 & -26.85 & 14.51 \\
\hline & IT & -12.936 & 5.292 & .978 & -39.87 & 14.00 \\
\hline & Pharmaceutics & 0.154 & 4.205 & 1.000 & -21.25 & 21.56 \\
\hline & Capital Goods & -2.923 & 4.205 & 1.000 & -24.33 & 18.48 \\
\hline & $\begin{array}{l}\text { Other Fin. } \\
\text { Services }\end{array}$ & 5.469 & 3.784 & 1.000 & -13.79 & 24.73 \\
\hline & Construction & -3.769 & 5.642 & 1.000 & -32.49 & 24.95 \\
\hline & $\begin{array}{l}\text { Transport } \\
\text { Services }\end{array}$ & 5.631 & 5.642 & 1.000 & -23.09 & 34.35 \\
\hline & $\begin{array}{l}\text { Food and } \\
\text { Beverages }\end{array}$ & 3.231 & 5.642 & 1.000 & -25.49 & 31.95 \\
\hline \multirow{11}{*}{$\begin{array}{l}\text { Commercial } \\
\text { Services }\end{array}$} & Automobiles & -7.800 & 5.980 & 1.000 & -38.24 & 22.64 \\
\hline & Chemicals & 0.978 & 5.980 & 1.000 & -29.46 & 31.41 \\
\hline & Telecom & -5.967 & 6.492 & 1.000 & -39.01 & 27.08 \\
\hline & $\begin{array}{l}\text { Consumer } \\
\text { Products }\end{array}$ & -15.200 & 5.537 & .937 & -43.38 & 12.98 \\
\hline & IT & -21.967 & 6.492 & .717 & -55.01 & 11.08 \\
\hline & Pharmaceutics & -8.877 & 5.642 & 1.000 & -37.59 & 19.84 \\
\hline & Capital Goods & -11.954 & 5.642 & .995 & -40.67 & 16.76 \\
\hline & $\begin{array}{l}\text { Other Fin. } \\
\text { Services }\end{array}$ & -3.562 & 5.335 & 1.000 & -30.72 & 23.59 \\
\hline & Construction & -12.800 & 6.781 & .999 & -47.31 & 21.71 \\
\hline & $\begin{array}{l}\text { Transport } \\
\text { Services }\end{array}$ & -3.400 & 6.781 & 1.000 & -37.91 & 31.11 \\
\hline & $\begin{array}{l}\text { Food and } \\
\text { Beverages }\end{array}$ & -5.800 & 6.781 & 1.000 & -40.31 & 28.71 \\
\hline \multirow[t]{10}{*}{ Automobiles } & Chemicals & 8.778 & 5.054 & 1.000 & -16.95 & 34.50 \\
\hline & Telecom & 1.833 & 5.651 & 1.000 & -26.93 & 30.59 \\
\hline & $\begin{array}{l}\text { Consumer } \\
\text { Products }\end{array}$ & -7.400 & 4.521 & 1.000 & -30.41 & 15.61 \\
\hline & IT & -14.167 & 5.651 & .972 & -42.93 & 14.59 \\
\hline & Pharmaceutics & -1.077 & 4.649 & 1.000 & -24.74 & 22.59 \\
\hline & Capital Goods & -4.154 & 4.649 & 1.000 & -27.82 & 19.51 \\
\hline & $\begin{array}{l}\text { Other Fin. } \\
\text { Services }\end{array}$ & 4.238 & 4.272 & 1.000 & -17.50 & 25.98 \\
\hline & Construction & -5.000 & 5.980 & 1.000 & -35.44 & 25.44 \\
\hline & $\begin{array}{l}\text { Transport } \\
\text { Services }\end{array}$ & 4.400 & 5.980 & 1.000 & -26.04 & 34.84 \\
\hline & $\begin{array}{l}\text { Food and } \\
\text { Beverages }\end{array}$ & 2.000 & 5.980 & 1.000 & -28.44 & 32.44 \\
\hline \multirow[t]{5}{*}{ Chemicals } & Telecom & -6.944 & 5.651 & 1.000 & -35.70 & 21.82 \\
\hline & $\begin{array}{l}\text { Consumer } \\
\text { Products }\end{array}$ & -16.178 & 4.521 & .617 & -39.19 & 6.83 \\
\hline & IT & -22.944 & 5.651 & .361 & -51.70 & 5.82 \\
\hline & Pharmaceutics & -9.855 & 4.649 & .995 & -33.52 & 13.81 \\
\hline & Capital Goods & -12.932 & 4.649 & .929 & -36.59 & 10.73 \\
\hline
\end{tabular}




\begin{tabular}{|c|c|c|c|c|c|c|}
\hline \multirow{12}{*}{ Telecom } & $\begin{array}{l}\text { Other Fin. } \\
\text { Services }\end{array}$ & -4.540 & 4.272 & 1.000 & -26.28 & 17.20 \\
\hline & Construction & -13.778 & 5.980 & .988 & -44.21 & 16.66 \\
\hline & $\begin{array}{l}\text { Transport } \\
\text { Services }\end{array}$ & -4.378 & 5.980 & 1.000 & -34.81 & 26.06 \\
\hline & $\begin{array}{l}\text { Food and } \\
\text { Beverages }\end{array}$ & -6.778 & 5.980 & 1.000 & -37.21 & 23.66 \\
\hline & $\begin{array}{l}\text { Consumer } \\
\text { Products }\end{array}$ & -9.233 & 5.179 & .999 & -35.59 & 17.13 \\
\hline & IT & -16.000 & 6.190 & .963 & -47.51 & 15.51 \\
\hline & Pharmaceutics & -2.910 & 5.292 & 1.000 & -29.84 & 24.02 \\
\hline & Capital Goods & -5.987 & 5.292 & 1.000 & -32.92 & 20.95 \\
\hline & $\begin{array}{l}\text { Other Fin. } \\
\text { Services } \\
\end{array}$ & 2.405 & 4.963 & 1.000 & -22.86 & 27.67 \\
\hline & Construction & -6.833 & 6.492 & 1.000 & -39.88 & 26.21 \\
\hline & $\begin{array}{l}\text { Transport } \\
\text { Services }\end{array}$ & 2.567 & 6.492 & 1.000 & -30.48 & 35.61 \\
\hline & $\begin{array}{l}\text { Food and } \\
\text { Beverages }\end{array}$ & 0.167 & 6.492 & 1.000 & -32.88 & 33.21 \\
\hline \multirow{7}{*}{$\begin{array}{l}\text { Consumer } \\
\text { Products }\end{array}$} & IT & -6.767 & 5.179 & 1.000 & -33.13 & 19.59 \\
\hline & Pharmaceutics & 6.323 & 4.063 & 1.000 & -14.35 & 27.00 \\
\hline & Capital Goods & 3.246 & 4.063 & 1.000 & -17.43 & 23.92 \\
\hline & $\begin{array}{l}\text { Other Fin. } \\
\text { Services }\end{array}$ & 11.638 & 3.624 & .795 & -6.81 & 30.09 \\
\hline & Construction & 2.400 & 5.537 & 1.000 & -25.78 & 30.58 \\
\hline & $\begin{array}{l}\text { Transport } \\
\text { Services }\end{array}$ & 11.800 & 5.537 & .995 & -16.38 & 39.98 \\
\hline & $\begin{array}{l}\text { Food and } \\
\text { Beverages }\end{array}$ & 9.400 & 5.537 & 1.000 & -18.78 & 37.58 \\
\hline \multirow[t]{6}{*}{ IT } & Pharmaceutics & 13.090 & 5.292 & .975 & -13.84 & 40.02 \\
\hline & Capital Goods & 10.013 & 5.292 & .999 & -16.92 & 36.95 \\
\hline & $\begin{array}{l}\text { Other Fin. } \\
\text { Services }\end{array}$ & 18.405 & 4.963 & .547 & -6.86 & 43.67 \\
\hline & Construction & 9.167 & 6.492 & 1.000 & -23.88 & 42.21 \\
\hline & \begin{tabular}{|l|} 
Transport \\
Services
\end{tabular} & 18.567 & 6.492 & .912 & -14.48 & 51.61 \\
\hline & $\begin{array}{l}\text { Food and } \\
\text { Beverages }\end{array}$ & 16.167 & 6.492 & .974 & -16.88 & 49.21 \\
\hline \multirow[t]{5}{*}{ Pharmaceutics } & Capital Goods & -3.077 & 4.205 & 1.000 & -24.48 & 18.33 \\
\hline & $\begin{array}{l}\text { Other Fin. } \\
\text { Services }\end{array}$ & 5.315 & 3.784 & 1.000 & -13.94 & 24.57 \\
\hline & Construction & -3.923 & 5.642 & 1.000 & -32.64 & 24.79 \\
\hline & $\begin{array}{l}\text { Transport } \\
\text { Services }\end{array}$ & 5.477 & 5.642 & 1.000 & -23.24 & 34.19 \\
\hline & $\begin{array}{l}\text { Food and } \\
\text { Beverages }\end{array}$ & 3.077 & 5.642 & 1.000 & -25.64 & 31.79 \\
\hline \multirow[t]{4}{*}{ Capital Goods } & $\begin{array}{l}\text { Other Fin. } \\
\text { Services }\end{array}$ & 8.392 & 3.784 & .992 & -10.87 & 27.65 \\
\hline & Construction & -0.846 & 5.642 & 1.000 & -29.56 & 27.87 \\
\hline & $\begin{array}{l}\text { Transport } \\
\text { Services }\end{array}$ & 8.554 & 5.642 & 1.000 & -20.16 & 37.27 \\
\hline & $\begin{array}{l}\text { Food and } \\
\text { Beverages }\end{array}$ & 6.154 & 5.642 & 1.000 & -22.56 & 34.87 \\
\hline
\end{tabular}




\begin{tabular}{|l|l|l|c|c|c|c|c|}
\hline & $\begin{array}{l}\text { Other Fin. } \\
\text { Services }\end{array}$ & Construction & -9.238 & 5.335 & 1.000 & -36.39 & 17.92 \\
\cline { 3 - 8 } & $\begin{array}{l}\text { Transport } \\
\text { Services }\end{array}$ & 0.162 & 5.335 & 1.000 & -26.99 & 27.32 \\
\cline { 2 - 7 } & $\begin{array}{l}\text { Food and } \\
\text { Ceverages }\end{array}$ & -2.238 & 5.335 & 1.000 & -29.39 & 24.92 \\
\cline { 2 - 7 } & $\begin{array}{l}\text { Transport } \\
\text { Services }\end{array}$ & 9.400 & 6.781 & 1.000 & -25.11 & 43.91 \\
\cline { 2 - 7 } & $\begin{array}{l}\text { Food and } \\
\text { Beverages }\end{array}$ & 7.000 & 6.781 & 1.000 & -27.51 & 41.51 \\
\cline { 2 - 8 } & $\begin{array}{l}\text { Food and } \\
\text { Transport } \\
\text { Services }\end{array}$ & -2.400 & 6.781 & 1.000 & -36.91 & 32.11 \\
\hline
\end{tabular}

Note: * The mean difference is significant at 0.05 levels.

Furthermore, Aly et al. (2010), found that formatting (presentation) of websites were superior in financial and communication industry of Egyptian companies. Turmin et al. (2016) reported a positive association between industry classification and corporate internet reporting. The results also consistent with studies conducted in India which found an association between industry type and internet reporting (Garg and Verma, 2010; Malhotra and Singh, 2005). The rejection of null hypothesis H0 allows the further analysis of pair wise comparison between the industries. Post hoc Scheffe's test (as samples are unequal) has conducted to examine how significant the corporate internet disclosure difference between each pair of industry type. The test result from Table 4 shows no significant difference between each pair of industry for the total score of corporate internet reporting. These findings suggest that companies in different industries in India have standardized web based corporate disclosure

\section{CONCLUSION}

The present paper is attempted to study the extent of internet reporting across different industry groups of listed Indian companies. The study reveals that among the 183 companies only one company does not have the website in this digital era. The internet reporting disclosure is not consistent among the different sectors. Companies in information technology industry are leading comparatively than other industries in the overall disclosure of content and presentation features. There is extensive variation among the sub-dimensions of content and presentation features such as banking and financial industry leading to the disclosure of financial information. Telecom industry has better customer support than other industry groups. The automobile industry is more concerned about the disclosure of corporate social responsibility because these units directly affect the environment. Only finance and telecom sectors are utilizing the E-commerce facility. These findings have supported the result of Aly et al. (2010) that communication and financial services industry are the determinants of voluntary disclosure. Of presentation dimension, Information technology industry is leading in the utilizing of multimedia and navigation for internet disclosure. All the sectors utilizing the presentation attribute such as file formats (PDF), link to social sites, downloading and printing of reports. Whereas, the result of a language translator, website update, browser compatibility is very disappointing with regards to all sectors. The results of ANOVA indicate that website content and presentation were significantly different across the industry groups. However, the multiple comparison test show no significant difference between each pair of industry for the total score of corporate web reporting.

The findings show that Indian companies are utilizing the internet technology for disseminating company's information. However, we observed numbers of significant content and presentation attributes being ignored by Indian companies. There 
is much scope for improvement in the disclosure regime. The policy makers of reporting companies identify the essential factors of internet disclosure, the establishment of separate information technology department wholly accountable for internet disclosure and training their employees 'how to compete with other sectors, locally and globally'. Moreover, regulatory bodies such as RBI, SEBI, IAS, etc. also take the initiative to assure the standardization, reliability, and completeness of the content \& presentation attributes of the website.

We acknowledge one of the most significant limitations of our study is the problem of generalization. Our result may not be representative of the companies in the other parts of the world due to different socio-economic culture, industrial structure and behavior.

\section{REFERENCES}

1) Abdelsalam, O., and Street, D., (2007), " An Examination of the Comprehensiveness of Corporate Internet Reporting Provided by London- Listed Companies". Journal of International Accounting Research". 6(2), 1-33.

2) Alali, F., \& Romero, S. (2012). The use of the Internet for corporate reporting in the Mercosur (Southern common market): The Argentina case. Advances in Accounting, 28(1), 157-167.

3) Aly, D., Simon, J. And Hussainey, K., (2010), “Determinants of Corporate Internet Reporting: Evidence from Egypt", Managerial Auditing Journal. 25(2), 182-202.

4) Bonsón, E. and Escobar, T., (2002), "A Survey on Voluntary Disclosure on the Internet. Empirica Evidence from 300 European Union Companies", The International Journal of Digital Accounting Research, 2(1), 27-51.

5) Craven, B. M. and Marston, C. L., (1999), "Financial reporting on the Internet by leading UK companies", The European Accounting Review, 8(2), 321-333.

6) Debreceny, R. and Gray, G.L., (1999), “Financial Reporting on the Internet and the External
Audit", European Accounting Review, 8(2), 335350.

7) Debreceny, R., Gray, G. L. And Rahman, A., (2002), "The determinants of Internet financial reporting", Journal of Accounting and Public Policy, 21(4-5), 371-394.

8) Despina, A. C., \& Demetrios, P. L. (2009). The web-based financial reporting adopted by the listed companies in the Athens Stock Exchange. Journal of Modern Accounting and Auditing, 5(7), 7.

9) Ettredge, M., Richardson, U.J. and Scholz, S., (2000), "Timely Financial Reporting at Corporate Websites," Communications of the ACM, 45(6), 67-71.

10) Garg, M.C. and Verma, D. V., (2010), “WebBased Corporate Reporting Practices in India", IUP Journal of Accounting Research and Audit Practices, 9(3), 7-19.

11) Ilias, A., Razak, M. Z. A., \& Rahman, R. A. (2015). The quality of non-Financial information on internet business reporting for Malaysian public listed companies (PLCS). International Journal of Business and Administrative Studies, 1(3), 1-11.

12) Kamel, H., \& Awadallah, E. (2017). The extent of voluntary corporate disclosure in the Egyptian Stock Exchange: Its determinants and consequences. Journal of Accounting in Emerging Economies, 7(2), 266-291.

13) Khan, M.N.A.A. and Ismail, N.A., (2012), “ An Empirical Study on Indexes of Internet Financial Reporting: The case of Malaysia", African Journal of Business Management, 6(5), 20862100.

14) Khan, M.N.A.A. and Omar, N.A.B., (2013), “ A Study of Importance Items of Internet Financial Reporting: A Case of Malaysian Auditors", Middle-East Journal of Scientific Research, 18(3), 340-351.

15) Larrán, M. and Giner, B., (2002), "The Use of the Internet for Corporate Reporting by Spanish Companies", The International Journal of Digital Accounting Research, 2(1), 53-82.

16) Louwers, T.J., Pasewark, W.R. and Typpo, E.W., 
(1996), "The Internet: Changing the Way Corporations Tell Their Story", CPA Journal, November, 24-28.

17) Lymer, A., (1999), "The Internet and the Future of Corporate Reporting in Europe", European Accounting Review, 8(2).

18) Marston, C., (2003), "Financial reporting on the Internet by leading Japanese companies", Corporate Communications: An International Journal, 8(1), 23-34.

19) Omran, M. A., \& Ramdhony, D. (2016). Determinants of internet financial reporting in African markets: the case of Mauritius. The Journal of Developing Areas, 50(4), 1-18.

20) Oyelere, P., Laswad, F. and Fisher, R., (2003),"Determinants of Internet Financial Reporting by New Zealand Companies", Journal of International Financial Management and Accounting, 14(1), 26-63.

21) Pertiwi, A.D. and Hermana, B., (2013), "Comparing Internet Financial Reporting Index between Bank and Non Bank in Indonesia", Journal of Internet Banking and Commerce, 18(2), 1-14.

22) Prabowo, R. and Angkoso, K.S., (2006), "Factors Influencing the Extent of Web-based Disclosure. An Empirical Analysis of Indonesian Manufacturing Firms", Journal Akuntansi Dan Keuangan, 8 (2), 92-98.

23) Raman, R., Gurdip, S., \& Mahesh, J. (2003). Reporting Financial Performance Through Internet: (A Case Study of Indian Corporate Sector). Management and Labour Studies, 28(4), 335-348.

24) Robbins, S.S. and Stylianou, A.C., (2003), "Global Corporate Web Sites : An Empirical Investigation of Content and Design. Information \& Management, 40(3), 205-212.

25) Turmin, S. Z., Hamid, F. A., \& Ghazali, N. A. M. (2016). Corporate Internet Reporting Within Malaysian Economic Sectors. International Journal of Economics \& Management, 10(2).

26) Uyar, A. (2011). Determinants of corporate reporting on the internet: An analysis of companies listed on the Istanbul Stock Exchange (ISE).Managerial Auditing Journal, 27(1), 87-104.

27) Wallace, R. O., Naser, K., \& Mora, A. (1994). The relationship between the comprehensiveness of corporate annual reports and firm characteristics in Spain. Accounting and business research, 25(97), 41-53.

28) Xiao, J. Z., Yang, H. and Chow, C. W. (2004), "The Determinants and Characteristics of Voluntary Internet-based Disclosures by Listed Chinese Companies", Journal of Accounting and Public Policy, 23(3), 191-225.

29) Xiao, Z., Sangster, A. and Dodgson, J.H., (1997), "The Relationship between Information Technology and Corporate Financial Reporting", Information Technology and People, 10(1), 11-30.

\section{Proceedings}

1) Khan, M.N.A.A. and Ismail, N.A., (2012), "Users's Opinions about the Importance of Internet Financial Reporting: Evidence From Malaysian Academics", Paper provided by Conference Master Resources in its series Annual Summit on Business and Entrepreneurial Studies (ASBES 2011) Proceeding with number 2012-023-162.

2) Lymer, A., (1997), "The Use of the Internet for Corporate Reporting. A discussion of the Issues and Survey of Current Usage in the UK", Paper presented at the 21st Annual Congress of the European Accounting Association, Antwerp, Belgium.

\section{Further reading}

1) Singh, M., (2009), "Corporate Disclosure Through Web: A Study of Selected Indian Companies", Ph.D Dissertation, Guru Nanak Dev University, Amritsar, Punjab, India. 


\section{Appendix}

\section{Distribution of Indian Companies across different Industry Groups}

\begin{tabular}{|l|l|}
\hline Sector & \multicolumn{1}{|c|}{ No. of Companies } \\
\hline Oil and Gas & 15 \\
\hline Metals and Mining & 11 \\
\hline Chemicals & 9 \\
\hline Construction Materials & 5 \\
\hline Commercial Services & 5 \\
\hline Capital Goods & 13 \\
\hline Utilities & 13 \\
\hline Automobiles & 9 \\
\hline Pharmaceuticals & 13 \\
\hline Telecom Services & 6 \\
\hline Transport Services & 5 \\
\hline Banking & 31 \\
\hline Other Financial Services & 21 \\
\hline Consumer Products & 16 \\
\hline Food and Beverages & 5 \\
\hline Information Technology & 6 \\
\hline Total & \\
\hline
\end{tabular}

